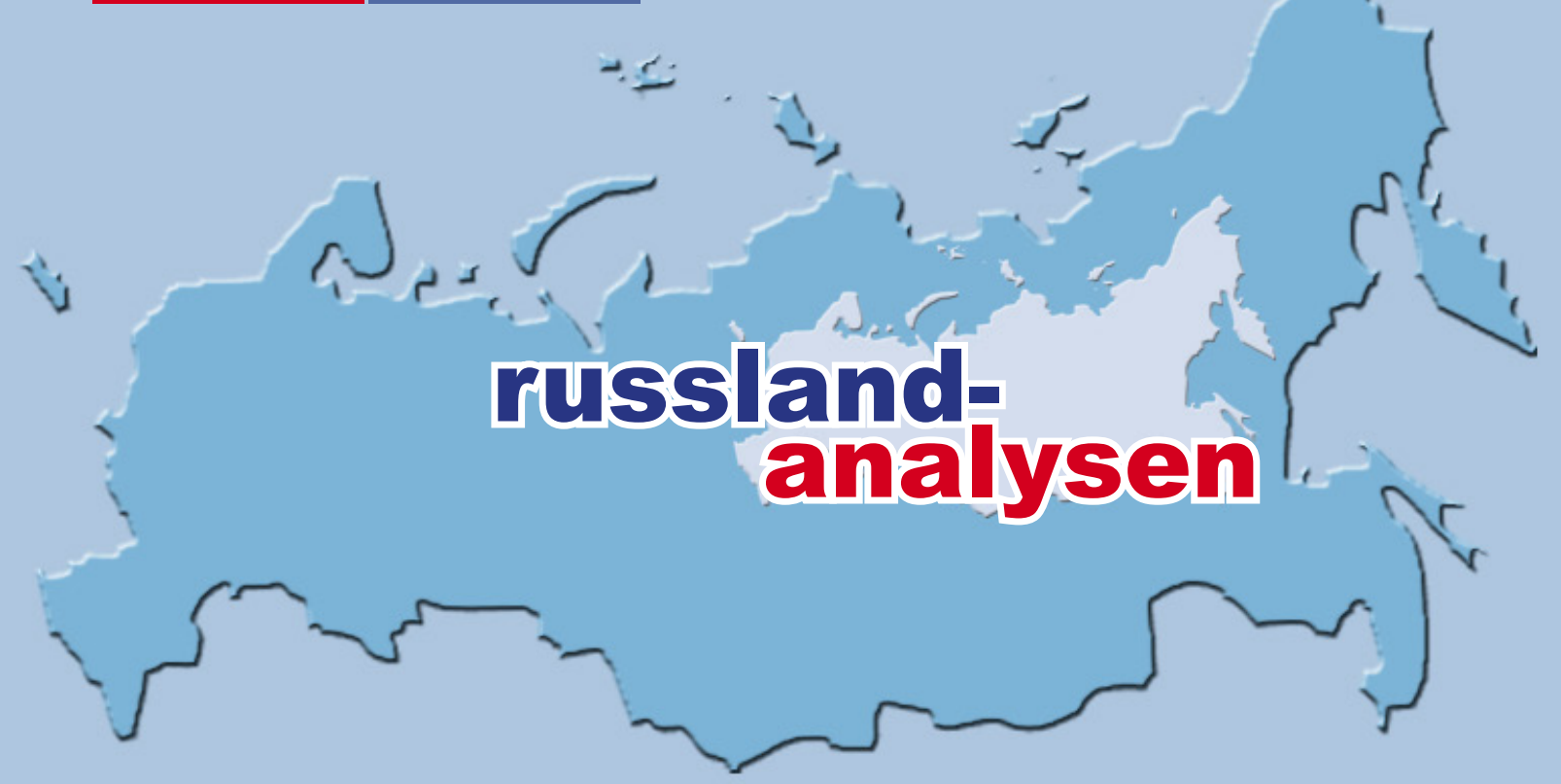

http://www.laender-analysen.de/russland/

\title{
RUSSLAND UND DIE TÜRKEI
}

\section{ANALYSE}

Krise der russisch-türkischen Beziehungen 2008-2015

Şener Aktürk, Istanbul

ANALYSE

Russisch-türkische Beziehungen: Bis auf Weiteres vollkommen in Trümmern

Toni Alaranta, Helsinki

STATISTIK

Die russisch-türkischen Beziehungen in Zahlen

UMFRAGE

Russen zu den Beziehungen zwischen der Türkei und Russland

- AUS RUSSISCHEN BLOGS

Zum Zerfall der "Demokratischen Koalition«

Sergey Medvedev, Berlin / Moskau

\section{NOTIZEN AUS MOSKAU}

Konzilianz und Härte - vom Verlust der Putinschen Stabilität

Jens Siegert, Moskau

UMFRAGE

Panama Papers

CHRONIK

22. April - 5. Mai 2016 


\title{
Krise der russisch-türkischen Beziehungen 2008-2015
}

\author{
Şener Aktürk, Istanbul
}

\section{Zusammenfassung}

Am 24. November schoss die Türkei einen russischen Kampfbomber vom Typ SU-24 ab, der den türkischen Luftraum verletzt hatte. Das ist als vorrangiger Grund für die nachfolgende spektakuläre Krise in den russisch-türkischen Beziehungen interpretiert worden. Dieser Zwischenfall sollte aber eher als Symptom, denn als Grund für eine erhebliche geopolitische Kehrtwende gesehen werden, die seit 2008 im Gange ist und in der sich Russland und die Türkei bei den militärischen Konflikten in Georgien 2008, in der Ukraine 2014 und in nun Syrien auf gegenüberliegenden Seiten wiederfanden.

\section{Der 24. November 2015: Symptom, aber nicht der Grund für die geopolitische Kehrtwende}

Während des Besuches des russischen Präsidenten Wladimir Putin in der Türkei am 1. Dezember 2014, der vor dem Hintergrund der westlichen Sanktionen gegen Russland stattfand, an denen sich die Türkei nicht beteiligte, dachte mancher, dass da eine russisch-türkische "Allianz« im Entstehen begriffen sei. Daher wirkte der Abschuss eines russischen Kampfbombers vom Typ SU-24, der den türkischen Luftraum verletzt hatte, wie ein Schock und wurde als vorrangiger Grund für die dramatischste Krise gesehen, die es in den russisch-türkischen Beziehungen im 21. Jahrhundert gegeben hat. Der Abschuss des russischen Kampfbombers war jedoch eher ein Symptom für die tektonischen Verwerfungen einer geopolitischen Dynamik, die früher Grundlage für die Zusammenarbeit der beiden Länder gewesen war.

Das beachtenswerteste Merkmal der Krise zwischen Russland und der Türkei ist, dass die beiden Länder weiterhin intensive wirtschaftliche Beziehungen unterhalten, was sich in einem jährlichen Handelsvolumen von 30 Milliarden US-Dollar in den Jahren 2011 bis 2014 zeigt, sowie in einem Abkommen über den Bau des ersten Atomkraftwerks der Türkei durch Russland im Jahre 2010 und in drei bis vier Millionen russischen Touristen, die alljährlich die Türkei besuchen [vgl. Grafiken 1-4 auf. S. 10-11]. Gleichwohl bestehen trotz prosperierender wirtschaftlicher Verbindungen fortgesetzt beträchtliche und sehr schwerwiegende geopolitische Interessenskonflikte mit Blick auf Georgien, die Ukraine und Syrien, wobei sich letzteres nach Russlands massiver Militärintervention, die am 1. September 2015 begann, zum Schauplatz eines eskalierenden Stellvertreterkrieges entwickelte.

\section{Das Vorspiel zum Bruch: Der "Fünftage-Krieg" zwischen Russland und Georgien im August 2008}

Georgien ist das Land, das für die Südkaukasus-Strategie der Türkei die Schlüsselrolle spielt, und es ist das einzige Land, das geographisch zwischen Russland und der Türkei liegt, gleichsam ein "Pufferstaat», der das Konfliktpotential zwischen den beiden Ländern mildert. Darüber hinaus ist Georgien seit der Wiedererlangung seiner staatlichen Unabhängigkeit im Jahr 1992 und insbesondere seit der Rosen-Revolution im November 2003, die Micheïl Saakaschwili im Januar 2004 als Präsidenten an die Macht brachte, ein enger Verbündeter der USA, der Türkei und Aserbaidschans gewesen. Georgien strebte unter Saakaschwili vehement einen Beitritt zur NATO an, ein Verlangen, das es der Türkei noch näher brachte, die ja aus georgischer Sicht der einzige Nachbar mit NATO-Mitgliedschaft ist. Die Pipeline Baku-Tbilissi-Ceyhan (BTC), ein wichtiges Ziel der türkischen Außenpolitik, das gemeinsame türkisch-aserbaidschanisch-georgische Interessen zementieren und kaspisches Öl für die Weltmärkte verfügbar machen sollte, wurde endlich fertiggestellt, und im Mai 2005 wurde das erste Öl aus Baku gepumpt und erreichte im Mai 2006 den türkischen Hafen Ceyhan. Neben anderen gemeinsamen Entwicklungsprojekten dieser Zeit gab es ein grundsätzliches Übereinkommen über den Bau der Eisenbahn Kars-Tbilissi, die die Eisenbahnnetze Georgiens, Aserbaidschans und der Türkei verbinden soll. Somit wurden spürbare und bedeutsame Schritte unternommen, um Georgien, Aserbaidschan und die Türkei einander näher zu bringen.

Der große Nachteil für Georgien war, dass es während der Rosen-Revolution über keines seiner autonomen Gebiete Abchasien, Adscharien und Südossetien eine de facto-Kontrolle hatte. Die Regierung Saakaschwili zwang im Mai 2004 Aslan Abaschidse, den prorussisch eingestellten Führer Adschariens, zum Rücktritt und brachte die Region unter die Kontrolle der georgischen Zentralregierung. Als Georgien jedoch im August 2008 versuchte, Südossetien unter seine Kontrolle zu bringen, erfolgte eine massive Antwort des russischen Militärs, bei der die georgische Armee in Südossetien besiegt und mehrere Städte im georgischen Kernland besetzt wurden. In der Folge des »FünftageKriegs", wie der Konflikt bald genannt wurde, erkannte Russland Abchasien und Südossetien als unabhängige 
Staaten an. Die entschiedene Niederlage Georgiens in diesem Konflikt, die Anerkennung der Unabhängigkeit von Abchasien und Südossetien durch Russland und die anschließende verstärkte militärische Präsenz Russlands in diesen Regionen, all das bedeutete einen schweren Schlag für den von der Türkei geführten Integrationsprozess im Kaukasus. Die Eisenbahnlinie Kars-Tbilissi ist derzeit noch nicht fertiggestellt, und es gelangten in Georgien 2012 ein neuer Ministerpräsident und 2013 dann ein neuer Präsident ins Amt, für den eine Versöhnung mit Russland Priorität hat. Ungeachtet des erheblichen Schadens, den ein russischer Sieg über Georgien für die türkischen Interessen bedeuten würde, gab es während des Krieges für Georgien keine aktive oder verbale Unterstützung durch die Türkei. Das konnte als ein Zeichen interpretiert werden, dass die Türkei ihre sehr viel wichtigeren Beziehungen zu Russland nicht wegen der Intervention in Georgien gefährden wollte.

\section{Zusammenarbeit trotz Konflikt: Handel und der AKW-Deal vom Mai 2010}

Nach dem Fünftagekrieg wurde die russisch-türkische Zusammenarbeit fortgesetzt und in einigen anderen Bereichen mit gemeinsamen Interessen sogar ausgebaut. Das wohl bemerkenswerteste Beispiel, berücksichtigt man seine wirtschaftliche Dimension sowie die strategische und symbolische Bedeutung, ist das russisch-türkische Abkommen zum Bau eines Atomkraftwerks. Das Abkommen wurde im Mai 2010 unterzeichnet, im Juli 2010 vom türkischen Parlament ratifiziert und sieht vor, dass ein Tochterunternehmen der russischen Staatskorporation »Rosatom « in Akkuyu an der türkischen Mittelmeerküste ein Atomkraftwerk bauen und betreiben wird.

Handel und Tourismus waren zwei weitere Bereiche intensiven russisch-türkischen Austauschs. Das bilaterale Handelsvolumen stieg von rund 23 Milliarden US-Dollar im Jahr 2009 auf 33 Milliarden US-Dollar im Jahr 2012 und betrug 2013 und 2014 immer noch über 30 Milliarden. Die Anzahl russischer Touristen, die die Türkei besuchten, stieg kontinuierlich, betrug 2010 über drei Millionen sowie 2014 über vier Millionen (Habibe Ozdal, Kerim Has: Türkiye Rusya: Derin Ayrışma (mı?), in: Analist, Nr. 60, Februar 2016, S. 40). Die Türkei war nach Deutschland der zweitgrößte Abnehmer von russischem Erdgas in Europa und die Zahl russischer Touristen wird nur von der deutscher Touristen übertroffen. Somit haben sowohl Deutschland, als auch Russland ihre Position als die beiden bedeutendsten Partner der Türkei gefestigt.

\section{Anfang vom Ende? Der arabische Frühling seit Dezember 2010}

Die Kette antiautoritärer Proteste und Aufstände, die im Dezember 2010 in Tunesien ihren Anfang nahm und als "arabischer Frühling « bekannt wurde, stellte, so ließe sich argumentieren, eine kritische Entwicklung innerhalb der geopolitischen Dynamik dar, die Russland und die Türkei in entscheidender Weise in eine Gegnerschaft zueinander brachte, und die letztendlich in die Konfrontation führte, am dramatischsten sichtbar im Herbst 2015 im Syrien-Konflikt. Diese Interpretation birgt jedoch einen gewissen Grad an Verzerrung durch die Rückschau. Zum einen, weil die nordafrikanischen Länder, in denen der arabische Frühling begann, nämlich Tunesien, Libyen und Ägypten, geographisch nicht in der Nähe der Türkei und Russlands liegen und nicht unmittelbar als Schlüsselländer für eine russisch-türkische Konkurrenz betrachtet werden. Zweitens hätte es ein wechselseitiges Entgegenkommen in Bezug auf die russischen und türkischen Präferenzen geben können, wenn die beiden Seiten eine solche Verständigung gesucht hätten. Dieses im Übrigen nachvollziehbare Szenario stellte sich nicht ein.

Mit zunehmendem Schwung der Proteste stellte sich die Türkei auf die Seite der revolutionären Bewegungen in Ägypten, Tunesien und - nach kürzerem Zögern auch in Libyen, während Russland sich resolut auf die Seite des später gestürzten libyischen Diktators Muammar al-Gaddafi und Abdel Fatach al-Sisis, jenes Militärdiktators, der Mohammed Mursi, den ersten demokratisch gewählten Präsidenten Ägyptens, aus dem Amt gejagt hatte. Während die Türkei die wohl weltweit wortstärkste Unterstützerin von Präsident Mursi und der Regierung der Muslimbrüder gewesen war, gehörte Russland zu den ausdrücklichsten Unterstützern von al-Sisis anschließender Militärdiktatur. Die russischen und türkischen Präferenzen erwiesen sich also selbst in den nordafrikanischen Ländern, wo der arabische Frühling begonnen hatte, als diametral gegenüberstehend.

\section{Entscheidende Wegscheide: Die Annexion der Krim durch Russland im März 2014}

Während die Regimewechsel, die gesellschaftlichen Konflikte oder eine Machtübernahme durch das Militär in Ägypten, Libyen oder Tunesien keine unmittelbare Gefahr für die nationale Sicherheit oder die territoriale Integrität Russlands oder der Türkei darstellten, hatten die bedeutsamen Entwicklungen, die die Ukraine seit November 2013 erfassten, unmittelbare Konsequenzen sowohl für jedes einzelne der beiden Länder, als auch für die russisch-türkischen Beziehungen im Besonderen. Der unmittelbare Grund für die Massenproteste, die auf dem Unabhängigkeitsplatz in Kiew einsetzten, war eine geopolitische Wahl: Die ukrainische Regierung unter Präsident Janukowytsch und Ministerpräsident Asarow hatte die Vorbereitungen für eine Unterzeichnung des Assoziationsabkommens mit der Europäischen 
Union zugunsten einer stärkeren Anbindung an Russland abgebrochen.

Diese bedeutsame und offensichtlich prorussische Entscheidung provozierte jene Menschen, die einen proeuropäischen Kurs der Ukraine bevorzugten, was von November 2013 bis Februar 2014 zu Massenprotesten führte, die - zusammen mit Janukowytschs Absetzung als Präsident durch das Parlament - diesen ins ostukrainische Charkiw und dann nach Russland fliehen ließen. Der Machtantritt einer erklärtermaßen prowestlichen Regierung in der Ukraine, den die russische Politik als einen "Staatsstreich" auffasste und als unrechtmäßig anprangerte, war der Vorwand für die umgehend in Gang gesetzte Besetzung der Krim im Februar 2014. Dieser folgte ein zweifelhaftes Referendum unter Besatzungsbedingungen, das im März 2014 angeblich eine Zustimmung der Bevölkerung zur Annektierung der Halbinsel durch Russland ergab.

Die Annexion der Krim bedeutete eine wichtige Wegscheide für die russisch-türkischen Beziehungen. Die Krim nimmt im Schwarzen Meer eine zentrale Position ein, ähnlich der Zyperns im Östlichen Mittelmeer. Bis zur Annexion der Krim hatte die Türkei im Schwarzen Meer eine maritime und strategische Überlegenheit genossen. Die Annexion der Halbinsel durch Russland hat das strategische Gleichgewicht im Schwarzen Meer dramatisch verändert und Russland zu einer aufkommenden Hegemoniemacht mit offensivem Potential gemacht, das alle Anrainerstaaten des Schwarzen Meeres bedroht, auch die Türkei. Wie ich es bereits am 6. März 2014 in einem Interview für die armenisch-türkische Zeitung "Agos" formuliert habe, ist Russland "mit der Annexion der Krim zur größten unmittelbaren militärischen Gefahr für die Türkei geworden«, erneut, wie schon zu Zeiten des Kalten Krieges und in den vergangenen zwei Jahrhunderten (Şener Aktürk: Kırım’ın işgaliyle, Rusya Türkiye'nin en buyuk tehdidi olur, in: Agos, 6. März 2014, S. 3.). Die Krimtataren, die angestammte Bevölkerung der Halbinsel, sind in der Geschichte von den Moskauer Regierungen verfolgt worden und stellten sich daher vehement der Annexion entgegen und boykottierten das Referendum. Während nach Jahrhunderten von Verfolgung und Deportationen eine Viertelmillion Tataren auf der Krim übriggeblieben sind, gibt es in der Türkei bis zu einer Million Nachfahren von Krimtataren, darunter auch in den akademischen, Wirtschafts- und intellektuellen Eliten. Es hat in der Türkei zahlreiche Proteste gegen die russische Annexion der Krim gegeben. Das Wiederentstehen einer militärischen Bedrohung durch Russland (mit der Annexion der Krim), hat eine geopolitische Dynamik umgekehrt, die seit dem Ende der Sowjetunion einen relativen Frieden und ein hohes
Niveau der Zusammenarbeit zwischen Russland und der Türkei bedeutet hatte.

\section{Verstärkte Zusammenarbeit trotz des Konfliktes: "Turkish Stream« im Dezember 2014}

Ungeachtet der scheinbar unversöhnlichen Differenzen zwischen Russland und der Türkei wegen der Krim, setzte sich die wirtschaftliche Zusammenarbeit unvermindert fort. Die Türkei beteiligte sich nicht an den westlichen Embargos, die nach der Krim-Annexion gegen Russland verhängt wurden, und profitierte somit von der Umlenkung einiger russischer Güter in die Türkei. Derweil hat im April 2015 der Bau des Atomkraftwerks in Akkuyu begonnen, wobei geplant ist, den ersten Reaktor bis 2020 betriebsfertig zu machen. Nach jahrelangen Diskussionen verkündete Putin bei seinem Staatsbesuch in der Türkei am 1. Dezember 2015 den Plan für ein neues Gaspipeline-Projekt namens »Turkish Stream«, das das "South Stream«-Projekt ersetzen soll, das nach den westlichen Sanktionen gegen Russland aufgegeben worden war. Die Verkündung des »Turkish Stream«-Projektes überraschte die meisten Beobachter und nährte überzogene Spekulationen zu einer entstehenden Achse Moskau-Ankara. Im Zusammenspiel mit der Entfremdung der Türkei von der westlichen Allianz meldeten sich einige Gruppen, wie etwa die türkischen Eurasier, die sich seit Jahren für eine russisch-türkische Achse als neue geopolitische Orientierung stark gemacht hatten, noch mehr zu Wort.

\section{Der letzte Strohhalm: Die russische Intervention in Syrien im September}

Die Türkei hat wie die Vereinigten Staaten und Frankreich verschiedene Oppositionsgruppen unterstützt, die seit 2011 gegen das Regime von Assad gekämpft haben und die meist um die »Freie Syrische Armee« gruppiert sind. Auch als "gemäßigte Opposition" bezeichnet, haben diese Gruppen ihre stärksten Bastionen in Nordwestsyrien, bei den Städten Aleppo und Idlib, ganz in der Nähe der türkischen Grenze. Nachdem die Umzingelung Aleppos durch die Regierungstruppen im Februar 2014 gescheitert war, erzielten diese Oppositionsgruppen schnelle und erhebliche Gewinne, und das AssadRegime schien im Frühling und Sommer 2015 allmählich zusammenzubrechen.

Syrien unter der Baath-Partei ist während des Kalten Krieges ein prosowjetisch ausgerichteter Staat gewesen. Der Marinestützpunkt Tartus in Syrien - geerbt von der Sowjetunion - ist der einzige russische Militärstützpunkt im Mittelmeer. Russland hat Syrien diplomatisch unterstützt, vor allem im Sicherheitsrat der Vereinten Nationen, insbesondere, indem es das Regime 
von Assad vor internationaler Kritik nach dessen Einsatz von Giftgas in Gouta im August 2013 schützte. Dennoch war die militärische Intervention Russlands in Syrien, die am 1. September 2015 begann, für viele Beobachter eine Überraschung. Die russische Luftwaffe begann, Stellungen der syrischen Oppositionskräfte zu bombardieren, von denen viele direkt von der Türkei unterstützt werden; im Laufe mehrerer Monate verletzte die russische Luftwaffe in zahlreichen Fällen den türkischen Luftraum, bis schließlich am 24. November ein russischer Kampfbomber vom Typ SU-24 von der Türkei abgeschossen wurde. Dieser Zwischenfall sollte als Ergebnis einer beträchtlichen geopolitischen Kehrtwende interpretiert werden, die wie oben skizziert seit 2008 im Gange war.

\section{Die nächste rote Linie: Russische militärische Unterstützung für die PKK und die PYD}

Die russisch-türkischen Beziehungen haben am 24. November 2015 einen Tiefpunkt erreicht, doch würde ich dem entgegen halten, dass die Beziehungen in den 1990er Jahren, während des Kalten Krieges und zwischen dem Zarenreich und dem Osmanischen Reich sehr viel schlechter waren. Der entscheidende Wendepunkt erfolgte Ende 1998, als Abdullah Öcalan, der Anführer der marxistisch-leninistischen »Kurdischen Arbei- terpartei« (PKK), die von der Türkei, der EU und den USA als Terrororganisation eingestuft wird, unter türkischem Druck gezwungen wurde, Syrien zu verlassen und nach Moskau zu fliehen, wo er politisches Asyl suchte. Am 4. November stimmte die russische Staatsduma für eine Asylgewährung. Ungeachtet dieses Votums lehnte die russische Regierung Öcalans Antrag ab und zwang ihn, Moskau zu verlassen. Das war der entscheidende Wendepunkt in den russisch-türkischen Beziehungen. Jahrzehntelang hatte Moskau zahlreiche Terrorgruppen und bewaffnete Aufstände gegen die Türkei unterstützt. Durch die Ablehnung von Öcalans Antrag signalisierte Moskau, dass es die territoriale Integrität der Türkei achten werde, indem es nicht Terrorismus und bewaffneten Aufstand unterstützt. Im Gegenzug signalisierte die Türkei, dass es keinen gegen Russland gerichteten Terrorismus oder bewaffneten Aufstand unterstützen werde, was angesichts der starken Aufstandsbewegung im Nordkaukasus in jener Zeit für Russland wichtig war. Eine russische Unterstützung für die PKK oder deren syrischer Schwesterorganisation PYD ist die nächste rote Linie in den Beziehungen, deren Übertreten zu einer weiteren Verschlechterung der Beziehungen führen könnte; sie könnten auf ein Niveau absinken, das es seit der Wende von 1998 nicht mehr gegeben hatte.

Übersetzung aus dem Englischen: Hartmut Schröder

\section{Über den Autor}

Şener Aktürk ist Associate Professor an der Fakultät für Internationale Beziehungen der Koç-Universität in Istanbul. Zu seinen Werken gehört "Regimes of Ethnicity and Nationhood in Germany, Russia, and Turkey» (erschienen 2012 bei Cambridge University Press).

\section{Lesetipps}

- Aktürk, Şener: Toward a Turkish-Russian Axis? Conflicts in Georgia, Syria, and Ukraine, and Cooperation over Nuclear Energy, in: Insight Turkey 16.2014, Nr. 4, S. 13-22.

- Aktürk, Şener: The Fourth Style of Politics: Eurasianism as a Pro-Russian Rethinking of Turkey's Geopolitical Identity, in: Turkish Studies 16.2015, Nr. 1, S. 54-79. 


\section{Russisch-türkische Beziehungen: Bis auf Weiteres vollkommen in Trümmern}

Toni Alaranta, Helsinki

\section{Zusammenfassung}

Während des vergangenen Jahrzehnts haben Russland und die Türkei eine formale strategische Allianz entwickelt, die auf intensiven wirtschaftlichen Bindungen, gemeinsamen Energieprojekten und einer von beiden geteilten Ablehnung einer Verwestlichung gründete. Darüber hinaus haben die Präsidenten Putin und Erdoğan eine persönliche Beziehung entwickelt, in der politische Differenzen stets heruntergespielt wurden. Nachdem die Türkei über Syrien ein russisches Kampfflugzeug abgeschossen hat, liegen die russisch-türkischen Beziehungen bis auf Weiteres in Trümmern. Obwohl wechselseitige wirtschaftliche Abhängigkeiten auf eine Normalisierung der Beziehungen hinweisen könnte, ist eine solche wegen der langfristig sich vollkommen gegenüberstehenden strategischen Ziele hinsichtlich Syrien und der in der russischen Führung bestehenden Wahrnehmung von Verrat und Erniedrigung nirgendwo in Sicht. Direkt gesagt, scheinen Russland und die Türkei jetzt am Rande eines Krieges zu stehen.

\section{Der Abschuss der russischen SU 24 und seine Auswirkungen}

Man kann sich nur schwer der Schlussfolgerung entziehen, dass die Phase der russisch-türkischen strategischen Allianz endgültig vorbei ist, nachdem die Türkei am 24. November 2015 über der syrisch-türkischen Grenze ein russisches Kampfflugzeug abgeschossen hat. Der Zwischenfall hat eine recht lebhafte Debatte darüber ausgelöst, welche Entscheidungsprozesse zu dem Abschuss geführt haben. Es wurde argumentiert, dass die Türkei, deren vorrangiges außenpolitisches Ziel seit 2011 die Vertreibung des Regimes von Baschar al-Assad in Syrien war, den Abschuss als Instrument nutzte, um die entstehende, gegen den Islamischen Staat gerichtete Koalition zwischen Russland und dem Westen aufzubrechen, die in nahezu jeder praktischen Hinsicht den Kampf gegen Assad völlig an den Rand drängt (s. Shlykov: The End of a Russian-Turkish 'Golden Age'...; i. d. Lesetipps). Die gleiche Interpretation wurde jüngst von Patrick Cockburn vorgelegt (s. Cockburn: Syrian civil war...; i. d. Lesetipps); er kommt zu dem Schluss, dass der Abschuss ein geplanter Hinterhalt der Türkei gewesen sei, um das russische Engagement im Syrien-Krieg teuer und unpopulär zu machen. Andererseits hat zumindest ein türkischer Experte argumentiert, dass der Zwischenfall mit dem Kampfjet in Wirklichkeit ein geplanter russischer Trick war, um die Türkei zu isolieren und die AKPRegierung dazu zu bringen, ihre vehemente Haltung gegen Assad aufzugeben (s. Gursel: Ankara falls into Moscow's trap; i. d. Lesetipps). Worin sich die meisten Kommentatoren einig zu sein scheinen, ist, dass für die Türkei als Ergebnis des Zwischenfalls letztendlich nicht nur der mehr oder weniger vollständige Bankrott der türkischen "Akteursrolle« in Syrien steht - diese ließe sich jetzt nur durch eine direkte militärische Einmischung wiederherstellen - sondern auch das abrupte Ende der ein Jahrzehnt lang erfolgten Annäherung mit Russland.

\section{Von strategischer Zusammenarbeit zu plötzlicher Feindschaft}

Nachdem sich Putin und Erdoğan im Dezember 2014 in Ankara in anscheinend herzlicher Weise getroffen hatten, wobei die russische Delegation von erheblichem Gewicht war, begann das Jahr 2015 mit der Erwartung, dass sich die heiklen, gleichzeitig jedoch gegenseitigen Nutzen bringenden Beziehungen fortsetzen und sogar weiterentwickeln würden, zumindest durch intensivierte Wirtschaftsbeziehungen, gemeinsame Energieprojekte und einen lösungsorientierten Dialog zu internationalen Konflikten (Tarasov, Stanislav: Türkiye ve Rusya, stratejik ittifak kuruyor, in: Sputnik Türkiye, 12. Januar 2015; <http://tr.sputniknews.com/ana liz/20150112/1013408602.html>). Es wurde weithin angenommen, dass die beiden Länder es geschafft haben, ihre offensichtlichen Streitigkeiten - insbesondere in Bezug auf die Ukraine-Krise, die Krimtataren und vor allem auf Syrien - einzuhegen. Über die starke wirtschaftliche Grundlage hinaus ist die Entwicklung der persönlichen Beziehung zwischen Putin und Erdoğan weithin beachtet und publizistisch aufgearbeitet worden, etwa mit Schlagzeilen westlicher Medien wie »Putin und Erdoğan sind füreinander geschaffen« (s. z. B. Tharoor: How Russia’s Putin...; i. d. Lesetipps).

In einem Sinne jedoch ist die russisch-türkische strategische Allianz diesem auf Individuen konzentrierten Kommunikationsmodus zum Opfer gefallen. Putin scheint außer sich gewesen zu sein, weil die Türkei nach dem Abschuss nicht sofort mit der russischen Führung kommunizierte, sondern sich stattdessen an ihre NATO-Verbündeten wandte. Darüber hinaus gibt 
es Anzeichen, die nahelegen, dass Putins wütende Stellungnahme "Die Türkei hat uns einen Dolchstoß versetzt." die wahren Gefühle eines Menschen ausdrückte, der sich von jemandem betrogen sieht, den er als persönlichen Freund betrachtet hat. Dieses ist vielleicht die wichtigste Komponente für jene Einschätzungen, die die Beziehungen als irreparabel betrachten, solange Putin und Erdoğan an der Spitze ihrer Länder stehen. Wie es der ehemalige FSB-Chef Sergej Stepaschin jüngst beschrieb, "vergisst Putin niemals jene, die ihn verraten oder beleidigt haben" (Sputnik Turkiye: Putin ihaneti asla affetmez, 9. Januar 2016. <http://tr.sputniknews. com/rusya/20160109/1020107141/Vladimir-PutinRusya.html >). Wenn diese beiden Narrative zusammengefügt werden, ergibt sich das Bild des russischen Präsidenten (wobei Putin Russland personifiziert), der niemals denjenigen vergisst, der ihm angeblich in den Rücken gefallen ist (Erdoğan, der die Türkei personifiziert).

Andererseits haben in der Türkei sowohl die allgemeine Öffentlichkeit, als auch die AKP-Staatselite jüngst zwei langfristige Reflexe an den Tag gelegt, die beide mit der traditionellen Konzeption von einem einheitlichen „Vaterland / „Vater Staat« (devlet baba) verbunden sind: Intern wird gegen die kurdische separatistische PKK vorgegangen und nach außen ist da der historische »Große Feind« Russland. In diesem Kontext ist es bemerkenswert, dass kein Tag vergeht, an dem nicht jemand aus der türkischen Führung behauptet, dass Russland direkt die PKK unterstützt (s. Munyar: Weapons seized...; i. d. Lesetipps). Es scheint, als seien alte Ängste vor russischem Expansionismus jetzt, nach der Syrien-Krise, wieder aus dem kollektiven Gedächtnis auf der Überholspur an die Oberfläche gelangt. Während die PKK also als wichtigste innere Bedrohung wahrgenommen wird, ist Russland nun die gefährlichste äußere Bedrohung (s. Hurriyet Daily News: Russia replaces...; i. d. Lesetipps). Die Konzeption einer nationalen Bedrohung der Türkei hauptsächlich anhand dieser beiden Akteure zu vermitteln, könnte allerdings langfristig höchst problematisch werden, zumindest dann, wenn sie eine sehr viel ernstere Wahrnehmung der Gefahr durch den Dschihad verhindern. Viele erfahrene Beobachter weisen darauf hin, dass es insbesondere nach dem Selbstmordanschlag durch den "Islamischen Staat"im Zentrum von Istanbul (12. Januar 2016) von vorrangiger Bedeutung sei, dass die Türkei die Beseitigung der Gefahr durch den IS zur Priorität macht (s. z. B. Taşpınar: Threat perception...; i. d. Lesetipps). Das ist allerdings nicht in Sicht.

\section{Geopolitische und geokulturelle Konfrontation}

In diesem Kontext ist es wichtig zu verstehen, dass die antiwestliche, proislamistische Position des AKP-
Regimes kein rhetorisches Mittel ist, sondern ein essentielles Element, durch das die Partei als politische Bewegung definiert wird. Eingedenk dieser Prämissen stellt sich die Frage nach den komplizierten Beziehungen mit Russland. Die antiwestliche Staatsideologie des Regimes in Moskau, die Bilder von einer alten und authentischen russisch-orthodoxen imperialen Zivilisation reproduziert, die gegen einen "unmoralischen« Westen kämpft, weist erstaunliche Ähnlichkeiten mit dem islamischkonservativen Projekt der AKP auf, mit dem diese den Staat transformiert. Das innertürkische Streben, den Staat von den "verabscheuungswürdigen kemalistischen Verwestlichern« zurückzuholen, hat auch eine Verlängerung ins Außenpolitische, der zufolge die Türkei dazu bestimmt ist, Anführer der sunnitisch-islamischen Welt zu werden. Die Vorstellung von einer Welt, die in alte Zivilisationen geteilt ist, die ihre derzeitige Manifestation in den Nationalstaaten Russland und Türkei erfahren, wird explizit in Ahmet Davutoğlus wegweisendem Werk »Strategic Depth" entwickelt, einem Buch, das einen immensen Einfluss auf die Außenpolitik der AKPÄra gehabt hat. Davutoğlu betrachtet in seinem Buch eindeutig nicht nur den Westen, sondern auch Russland als »natürliche« Gegner der Türkei, während Länder mit einer weit in die Geschichte zurückreichenden imperialen Vergangenheit, etwa Syrien, zweitrangige Staaten und Akteure sind (Davutoğlu, Ahmet: Stratejik Derinlik. Turkiye'nin Uluslararası Konumu. Istanbul: Kure Yayınları 2001). Folgt man diesen Annahmen, ließe sich argumentieren, dass Andrej Kortunows Einschätzung, der zufolge die jüngste »strategische Allianz« zwischen Russland und der Türkei stets eher rhetorisch denn real gewesen sei, auch aus türkischer Perspektive einen Sinn ergibt. Die beiden Länder haben zwar intensive wirtschaftliche Beziehungen entwickelt, sich auf gemeinsame Energieprojekte geeinigt und ihre jeweils antiwestlichen Agenden für die eigenen Zwecke eingesetzt, betrachten einander aber angesichts ihrer jahrhundertealten Rivalität letztendlich als »unnatürliche Partner«.

Natürlich gibt es eine Reihe von Drittländern, die die zunehmenden russisch-türkischen Animositäten als erhebliche Störung wahrnehmen. Neben der NATO, die die Türkei und Russland wiederholt zu einer Deeskalierung ihres jüngsten Konfliktes aufgerufen hat, meldeten sich eine ganze Reihe postsowjetischer Staaten (angefangen von Kasachstan und Turkmenistan bis hin zu Georgien und Aserbaidschan) zu Wort und äußerten ihre Befürchtungen hinsichtlich der zunehmenden Spannungen im Kaukasus und in Zentralasien, und unterstrichen dabei, dass die Türkei und Russland so schnell wie möglich ihr ehemals gutes Verhältnis wiederherstellen sollten (s. Gottesman: The Caspian States...; 
i. d. Lesetipps). Diese Aufrufe, die an eine Vorstellung von "Verantwortung« appellieren, waren jedoch nicht hinreichend, um den Lauf der Ereignisse zu ändern.

Die Türkei ist bekanntermaßen stark von russischen Energieträgern (Erdgas) abhängig und hat nun eine vehemente Kampagne für alternative Energiequellen gestartet, wobei insbesondere versucht wird, den Umfang der Gaslieferungen aus Aserbaidschan zu erhöhen (s. Jones / Safarova: Turkey Hunts ...; i. d. Lesetipps). Andererseits braucht Russland die Türkei als wichtigen Energiekunden. Es gibt dennoch keinen Zweifel, dass beide Länder sich auf eine lange Zeit der Animosität einrichten; diese Einschätzung erhielt nach einem weiteren Zwischenfall noch mehr Nahrung: am 29. Januar erklärte das türkische Außenministerium, dass es erneut eine Verletzung des Luftraums durch Russland gegeben habe. Dieser Zwischenfall wurde von den türkischen Medien intensiv aufgegriffen, wobei Präsident Erdoğan verkündete, dass Russland "sich genötigt sehen werde, die Konsequenzen [solcher Aktionen] zu ertragen« (s. Today's Zaman: Turkish-Russian tension...; i. d. Lesetipps). Solche Stellungnahmen wurden durch Erklärungen von Präsident Erdoğan begleitet, dass er vergeblich versucht habe, Präsident Putin zu erreichen. Auf russischer Seite wurde dies als reiner Bluff bewertet. Der Duma-Abgeordnete Leonid Kalaschnikow meinte beispielsweise, dass der Vorwurf einer weiteren Luftraumverletzung lediglich ein verzweifelter Versuch Präsident Erdoğans sei, den Kontakt zu Präsident Putin wiederherzustellen (Cumhuriyet: Rus milletvekili. Erdoğan, Putin'le goruşebilmek icin her yolu deniyor!, 31. Januar 2016; <http://www. cumhuriyet.com.tr/haber/dunya/472991/Rus_millet vekili__Erdogan__Putin_le_goruse bilmek_icin_her yolu_deniyor_.html >).

\section{Keine Aussichten auf eine Wiederherstellung der russisch-türkischen Zusammenarbeit}

Bei der Betrachtung von all dem stellt sich allgemein das Gefühl ein, dass die türkische Führung recht verzweifelt darum bemüht ist, Kanäle zu finden, über die sie eine Wiederherstellung der Beziehungen zu Russland beginnen könnte, während gleichzeitig Präsident Erdoğan nicht die auf einen Regime Change in Syrien gerichtete Politik aufgeben wird, wie auch die Türkei die von Russland gestellten formalen Bedingungen für eine Normalisierung nicht akzeptieren wird. Also eine offizielle Entschuldigung und Entschädigung. Andererseits besitzt die russische Führung eine Weltsicht, in der das gewandelte Bild von Russland als Großmacht und somit als einem der Akteure, der nun in beträchtlichen Maße die allgemeinen Züge der internationalen Politik definiert, ihre außenpolitischen Initiativen bestimmen, sei es nun in der Ukraine oder in Syrien. In diesem Kontext kann das Bild, dass Russland durch die Türkei öffentlich bloßgestellt wurde (immerhin bejubelten Ukrainer den Abschuss und meinten, sie sollten das Gleich tun), nicht akzeptabel sein, was auch der Grund dafür ist, dass eine formale Entschuldigung durch die Türkei eine Vorbedingung für irgendeine weitere Zusammenarbeit ist. Des Weiteren ist die Ansicht, dass die Vorwürfe über eine weitere Verletzung des türkischen Luftraums durch Russland schlichtweg ein Versuch sind, in der Syrien-Frage eine Annäherung zwischen dem Westen und Russland zu verhindern, mittlerweile in der russischen Führung fest verwurzelt.

Vor diesem Hintergrund wird die türkische Entschlossenheit, weiterhin (zusammen mit Saudi-Arabien) die bewaffnete Opposition gegen das Regime in Syrien zu unterstützen, wie sie vor kurzem von Ministerpräsident Ahmet Davutoğlu bekräftigt wurde (Daily Sabah: Turkey to continue...; i. d. Lesetipps), jedweder Annäherung zwischen Russland und der Türkei im Wege stehen. Mehr noch: Wie der US-amerikanische Vizepräsident Joe Biden sogar ausdrücklich meinte, hat die Türkei weniger die "Gemäßigten« finanziert, als Hunderte Millionen Dollar und Tausende Tonnen Waffen in die Unterstützung von Dschabhat al-Nusra (al-Kaïda) und anderer extremistischer Dschihad-Elemente aus allen Teilen der Welt gepumpt (s. Al Monitor: Erdogan's slow turnaround...; i. d. Lesetipps). Die russische Führung weiß das nur zu gut und macht davon in ihrer eigenen Kriegspropaganda ausgiebig Gebrauch. Unter diesen Umständen ist es, anders gesagt, für Russland sehr einfach, sich als wohlmeinender Akteur und Kämpfer gegen den internationalen Dschihad-Terrorismus in Syrien darzustellen, während es selbst mindestens genauso entschlossen ist sicherzustellen, dass sein einziger Verbündeter im Nahen Osten, nämlich das Assad-Regime, sich hält. Während gleichzeitig die Türkei beharrlich versucht, ihre gegen Assad gerichtete Haltung beizubehalten, sehen einige prominente Kommentatoren nun Russland und die Türkei am Rande eines Krieges, da beide Parteien an der türkisch-syrischen Grenze Truppen zusammenziehen (s. z. B. Idiz: Will Turkey risk...; i. d. Lesetipps).

\section{Schlussfolgerungen}

Wechselseitige wirtschaftliche Abhängigkeit, gemeinsame Energieprojekte und ähnliche konservative autoritäre innenpolitische Projekte, die in den beiden Ländern verfolgt werden, scheinen alle auf eine umgehende Reparatur der russisch-türkischen Beziehungen hinzuweisen. Gleichwohl ist eine solche Normalisierung nirgendwo in Sicht. Im Gegenteil: Die völlig gegensätzliche strategische Ausrichtung in Syrien und der Standpunkt der derzeitigen russischen Führung, dass es nicht mög- 
lich ist, der Türkei ihr erniedrigendes Vorgehen (den Abschuss des russischen Kampfets) zu verzeihen, und das in einer Zeit, da es für Russland prioritär sei, sein Image als Großmacht wiederherzustellen, schafft eine konfliktgeladene Entwicklungsbahn, auf der die russisch-türkischen Beziehung über Jahre hinweg angespannt bleiben werden.

Übersetzung aus dem Englischen: Hartmut Schröder

\section{Über den Autor}

Toni Alaranta wurde 2012 an der Universität Helsinki in Gesellschaftswissenschaften promoviert. Gegenwärtig ist er Senior Research Fellow am Finnish Institute of International Affairs. Sein wichtigstes Interessengebiet ist türkische Politik und Geschichte. Nach der Promotion verfolgte er seine Studien an der Middle East Technical University in Ankara und der Universität Tallinn. Zu seinen jüngsten Werken über die Türkei gehören »National and State Identity in Turkey: The Transformation of the Republic's Status in the International System " (2015) und "Contemporary Kemalism: From Universal Secular-Humanism to Extreme Turkish Nationalism« (2014).

\section{Lesetipps}

- Al Monitor: Erdogan's slow turnaround on foreign fighters in Syria, 5. Oktober 2014; <http://www.al-monitor. com/pulse/originals/2014/10/turkey-erdogan-foreign-fighters.html\#ixzz3yNOAWlqU>.

- Cockburn, Patrick: Syrian civil war: Could Turkey be gambling on an invasion?, in: The Independent, 31. Januar 2016; <http://www.independent.co.uk/news/world/middle-east/syrian-civil-war-could-turkey-be-gambling-onan-invasion-a6844171.html>.

- Cumhuriyet: Rus milletvekili: Erdoğan, Putin’le goruşebilmek icin her yolu deniyor!, 31. January 2016; < http:// www.cumhuriyet.com.tr/haber/dunya/472991/Rus_milletvekili__Erdogan__Putin_le_gorusebilmek_icin_her_ yolu_deniyor_html>.

- Daily Sabah: Turkey to continue siding with Syrian people against regime, Daesh, foreign powers aiming occupation, 1. Februar 2016; <http://www.dailysabah.com/diplomacy/2016/01/31/turkey-to-continue-siding-with -syrian-people-against-regime-daesh-foreign-powers-aiming-occupation>.

- Gottesman, Ewan: The Caspian States in Russia’s Military Bind, in: The Diplomat, 27. November 2015; < http:// thediplomat.com/2015/11/turkey-russia-tensions-put-caspian-states-in-a-bind/>.

- Gursel, Kadri: Ankara falls into Moscow's trap,in: Al Monitor, 9. Dezember 2015; <http://www.al-monitor.com/ pulse/originals/2015/12/turkey-russia-syria-downing-of-russian-jet-moscow-lure-trap.html>.

- Hurriyet Daily News: Russia replaces Israel at top of threat list for Turks: Survey, 13. Januar 2016; <http://www.hurriyetdailynews.com/russia-replaces-israel-at-top-of--threat-list-for-turks-survey. aspx?PageID=238\&NID=93808\&NewsCatID=338> .

- Idiz, Semih: Will Turkey risk military confrontation with Russia?, in: Al Monitor, 2. Februar 2016; <http://www. al-monitor.com/pulse/originals/2016/02/turkey-russia-moscow-tries-to-draw-ankara-into-fight.html>.

- Jones, Dorian; Durna Safarova: Turkey Hunts for Alternatives to Russian Energy, in: EurasiaNet, 27. Januar 2016; $<$ http://www.eurasianet.org/node/77016>.

- Kortunov, Andrey: The Russian-Turkish Crisis: a Deficit of the Strategic Depth, erschienen bei: Russian International Affairs Council, 4. Januar 2016; <http://russiancouncil.ru/en/inner/?id_4=7064\#top-content >.

- Munyar, Vahap: Weapons seized in southeast Russian-made: Turkish president, in: Hurriyet Daily News, 2. Februar 2016; <http://www.hurriyetdailynews.com/weapons-seized-in-southeast-russian-made-turkish-presi dent.aspx?pageID=238\&nID=94629\&NewsCatID=338>.

- Shlykov, Pavel: The End of a Russian-Turkish 'Golden Age', Carnegie Moscow Center, 25. November 2015; < http:// carnegie.ru/commentary/2015/11/25/end-of-russian-turkish-golden-age/imca>.

- Taşpınar, Omer: Threat perception in Turkey needs to change, in: Today's Zaman, 13. Januar 2016; <http://www. todayszaman.com/columnist/omer-taspinar/threat-perception-in-turkey-needs-to-change_409558.html>.

- Tharoor, Ishaan: How Russia’s Putin and Turkey's Erdogan were made for each other, in: The Washington Post, 2. December 2014; <https://www.washingtonpost.com/news/worldviews/wp/2014/12/02/how -russias-putin-and-turkeys-erdogan-were-made-for-each-other/>.

- Today's Zaman: Turkish-Russian tension flares anew as Ankara warns Moscow over airspace violation, 31. Januar 2016; <http://www.todayszaman.com/diplomacy_turkish-russian-tension-flares-anew-as-ankarawarns-mosco w-over-airspace-violation_411062.html>. 


\section{Die russisch-türkischen Beziehungen in Zahlen}

Grafik 1: Wichtigste Handelspartner der Türkei: Exporte

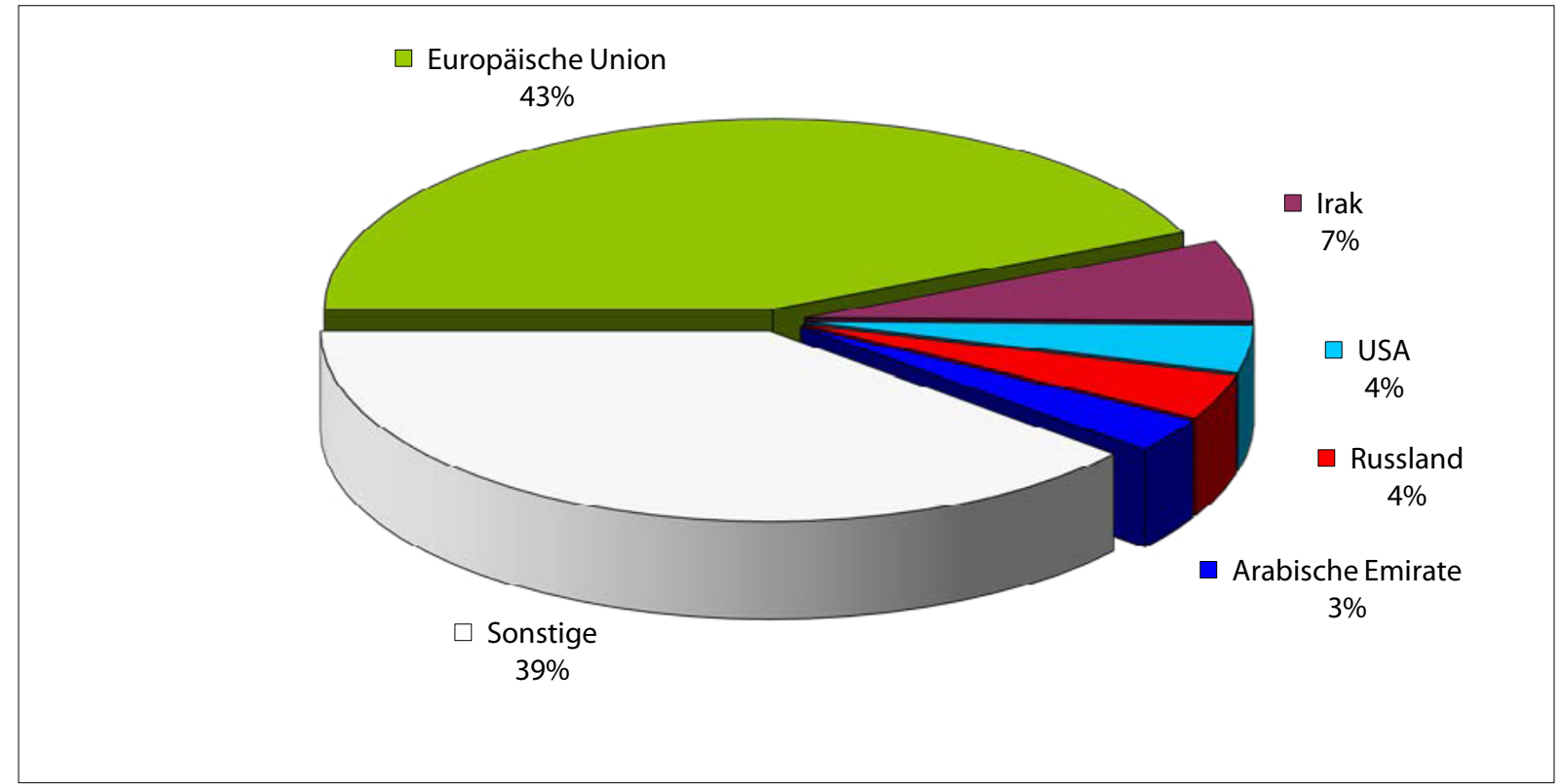

Quelle: Angaben der Welthandelsorganisation WTO, <http://stat.wto.org/CountryProfile/WSDBCountryPFView.aspx?Language= E\&Country=RU\%2cTR>, September 2015

Grafik 2: Wichtigste Handelspartner der Türkei: Importe

$\square$ Europäische Union

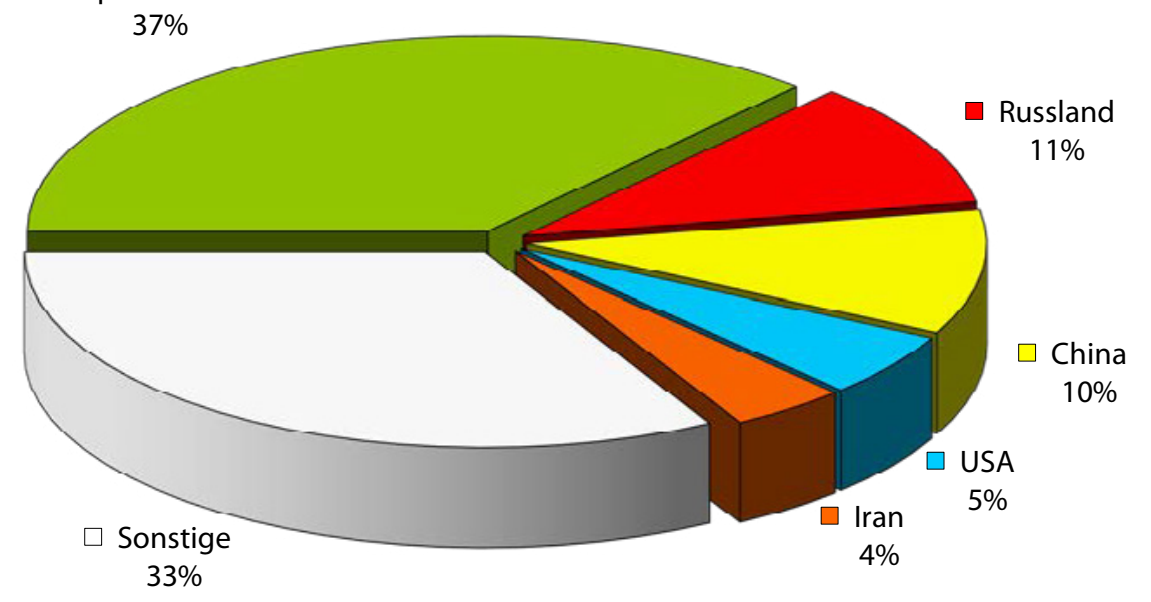

Quelle: Angaben der Welthandelsorganisation WTO, <http://stat.wto.org/CountryProfile/WSDBCountryPFView.aspx?Language= E\&Country=RU\%2cTR $>$, September 2015 


\section{Grafik 3: Außenhandel Russland in Mio. US-Dollar}

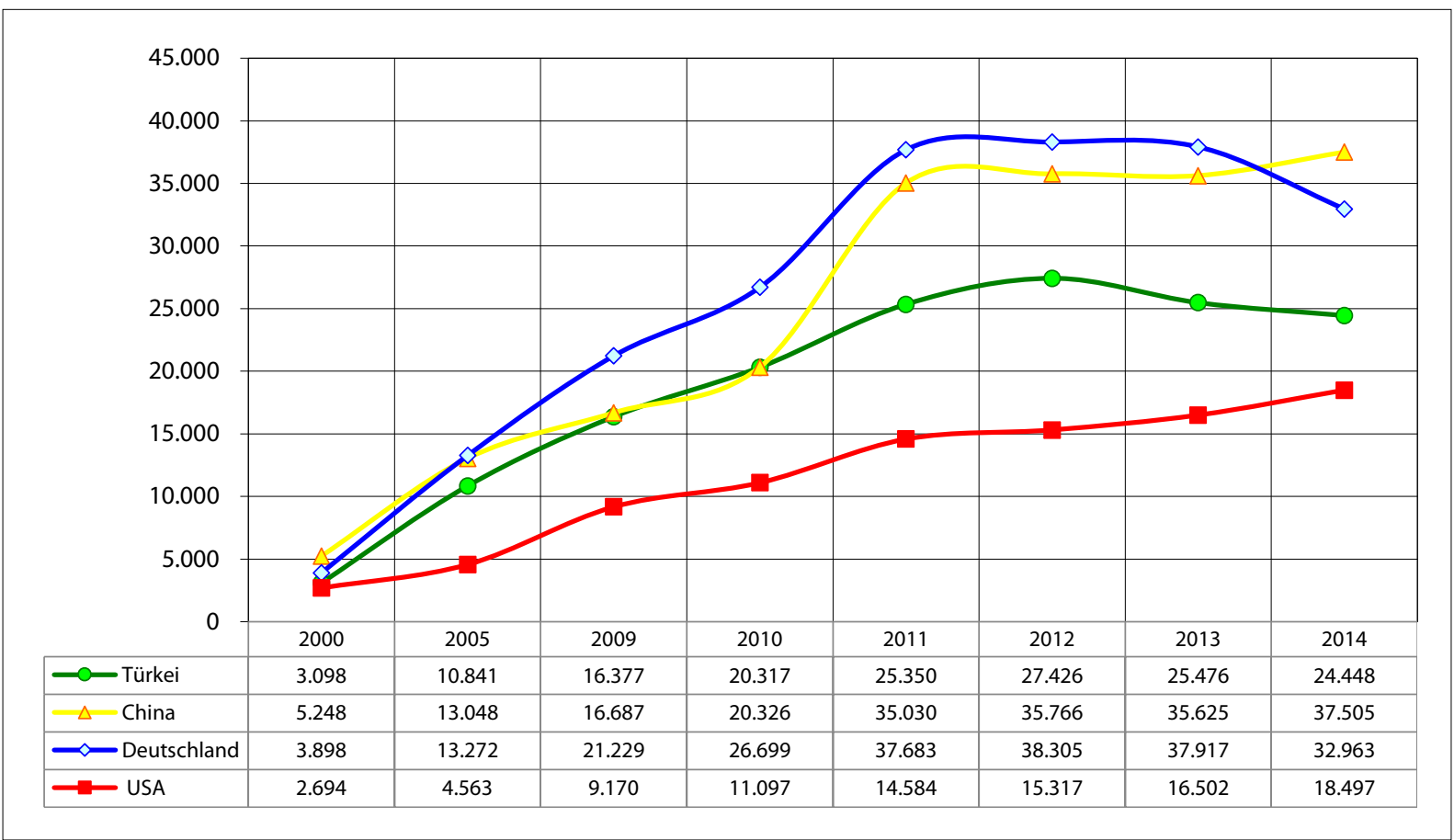

Quelle: Angaben der russischen Statistikbehörde Rosstat, <http://www.gks.ru/bgd/regl/b15_12/IssWWW.exe/stg/d02/27-06.htm>

\section{Grafik 4: Anzahl touristischer Reisen zwischen Russland und der Türkei}

Anzahl touristischer Reisen in die Türkei aus Russland

Anzahl touristischer Reisen aus der Türkei nach Russland

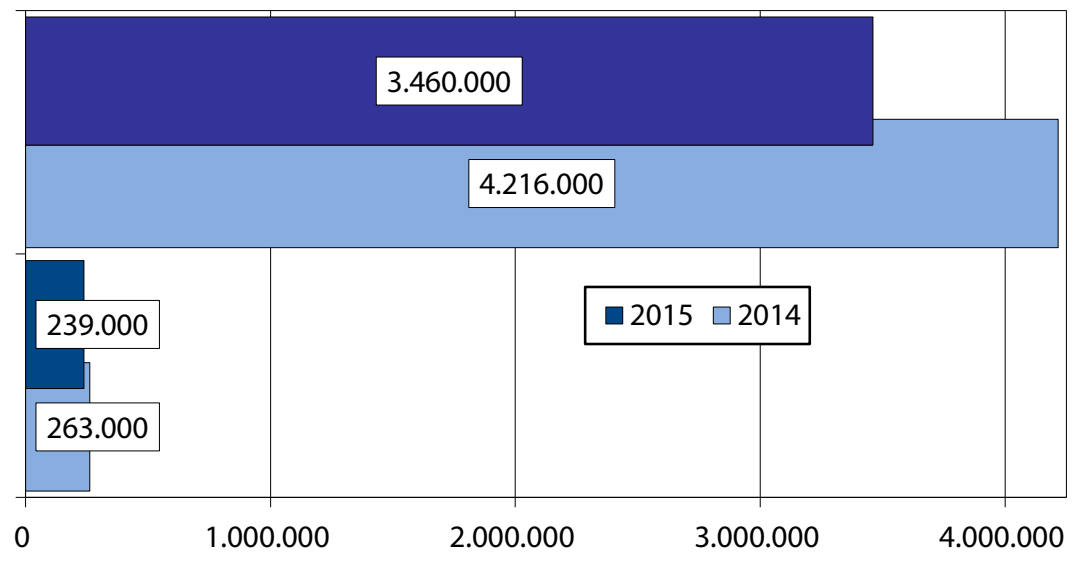

Quelle: Angaben der russischen Statistikbehörde Rosstat <http://www.gks.ru/wps/wcm/connect/rosstat_main/rosstat/ru/statistics/ enterprise/retail/\#> 


\section{Russen zu den Beziehungen zwischen der Türkei und Russland}

Grafik 5: Wie schätzen Sie die derzeitigen Beziehungen zwischen Russland und der Türkei ein?

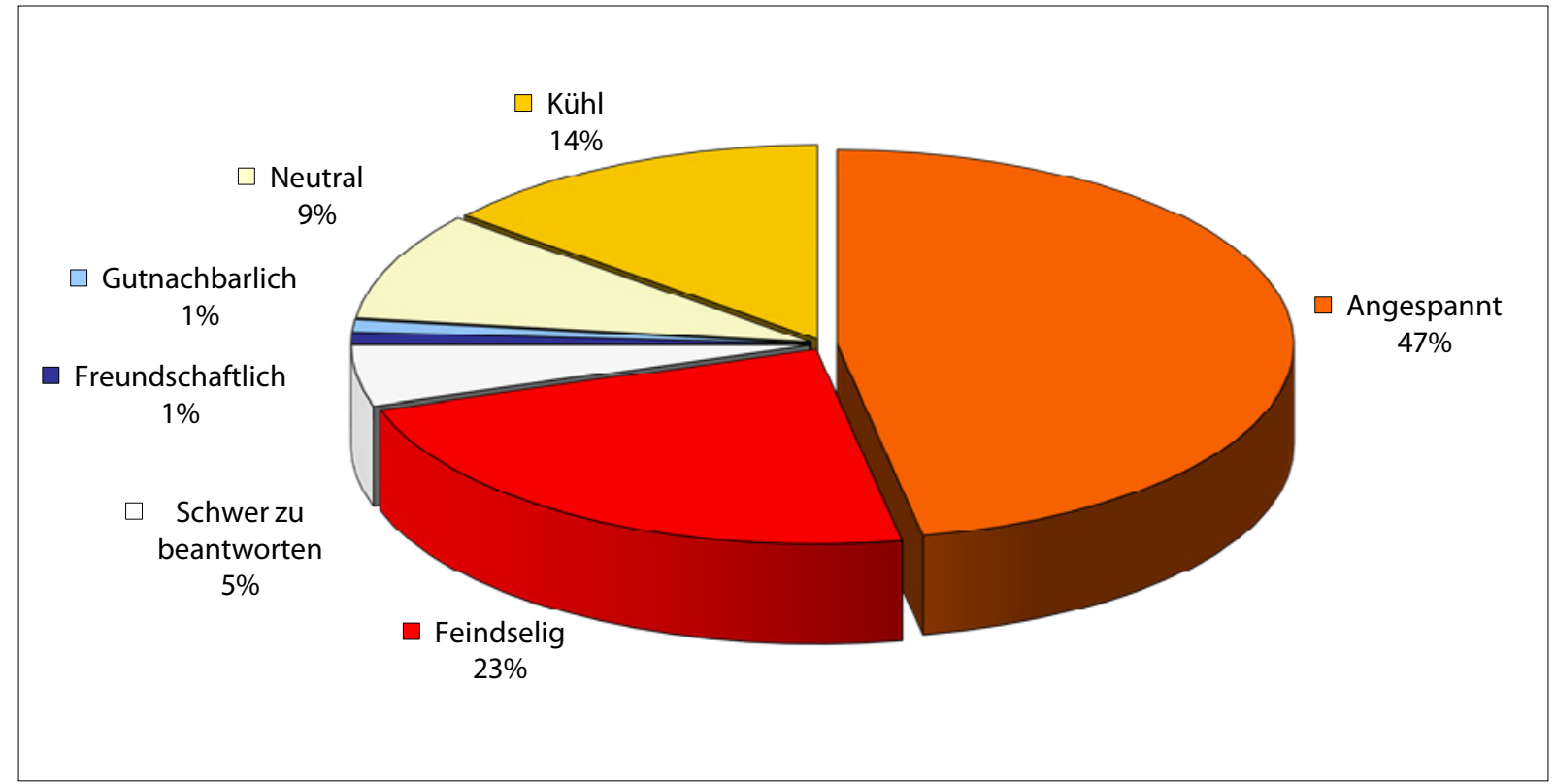

Quelle: Umfragen des WZIOM vom 6.-7. Februar 2016, <http://wciom.ru/index.php?id=236\&uid=115585>, 18. Februar 2016

Grafik 6: In welche Richtung werden sich die Beziehungen zwischen Russland und der Türkei in der nächsten Zeit entwickeln?

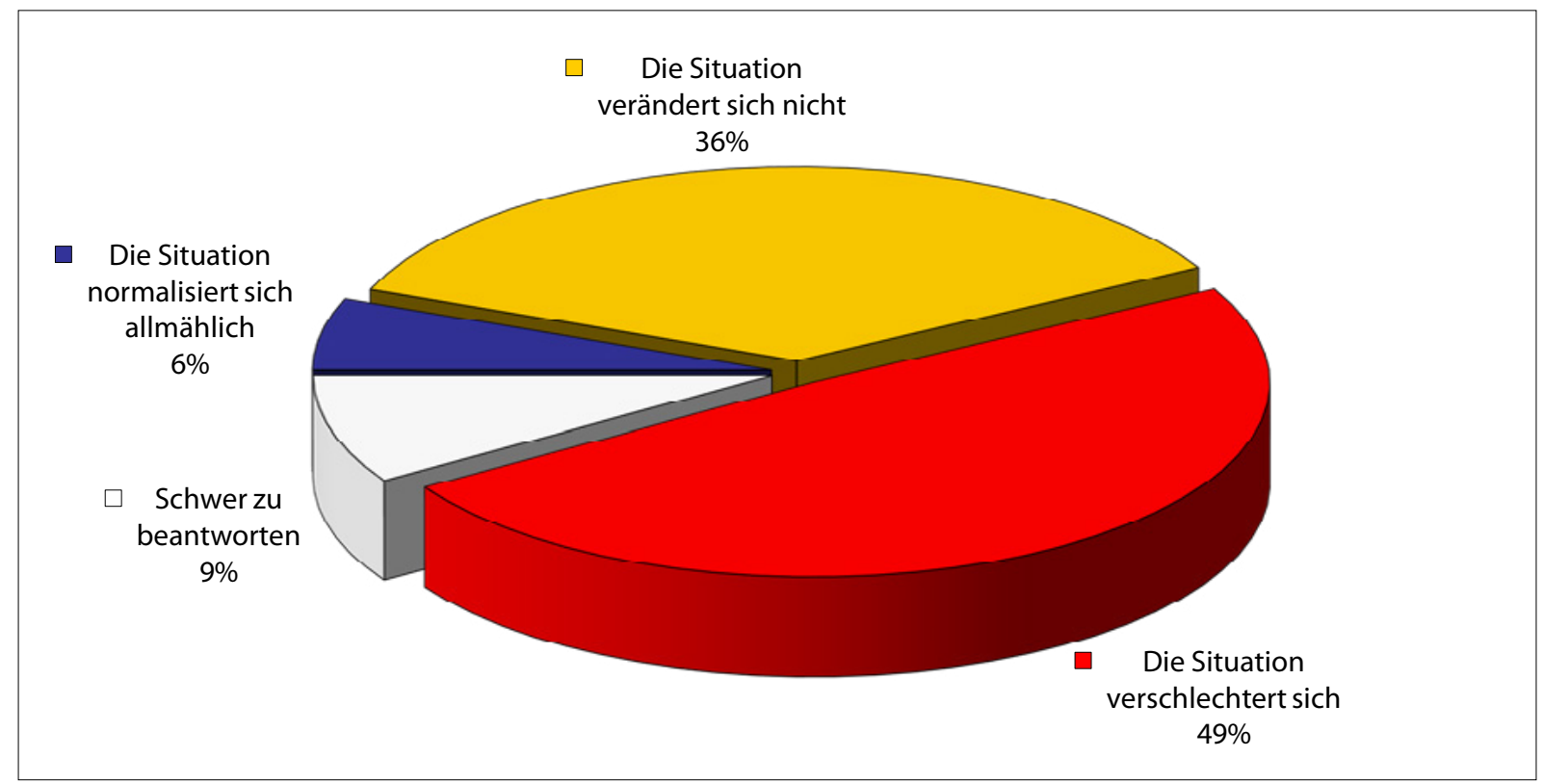

Quelle: Umfragen des WZIOM vom 6.-7. Februar 2016, <http://wciom.ru/index.php?id=236\&uid=115585>, 18. Februar 2016 
Grafik 7: Wer trägt in erster Linie die Schuld daran, das ein russisches Kampfflugzeug im Einsatz im Syrienkrieg durch das türkische Militär abgeschossen wurde?

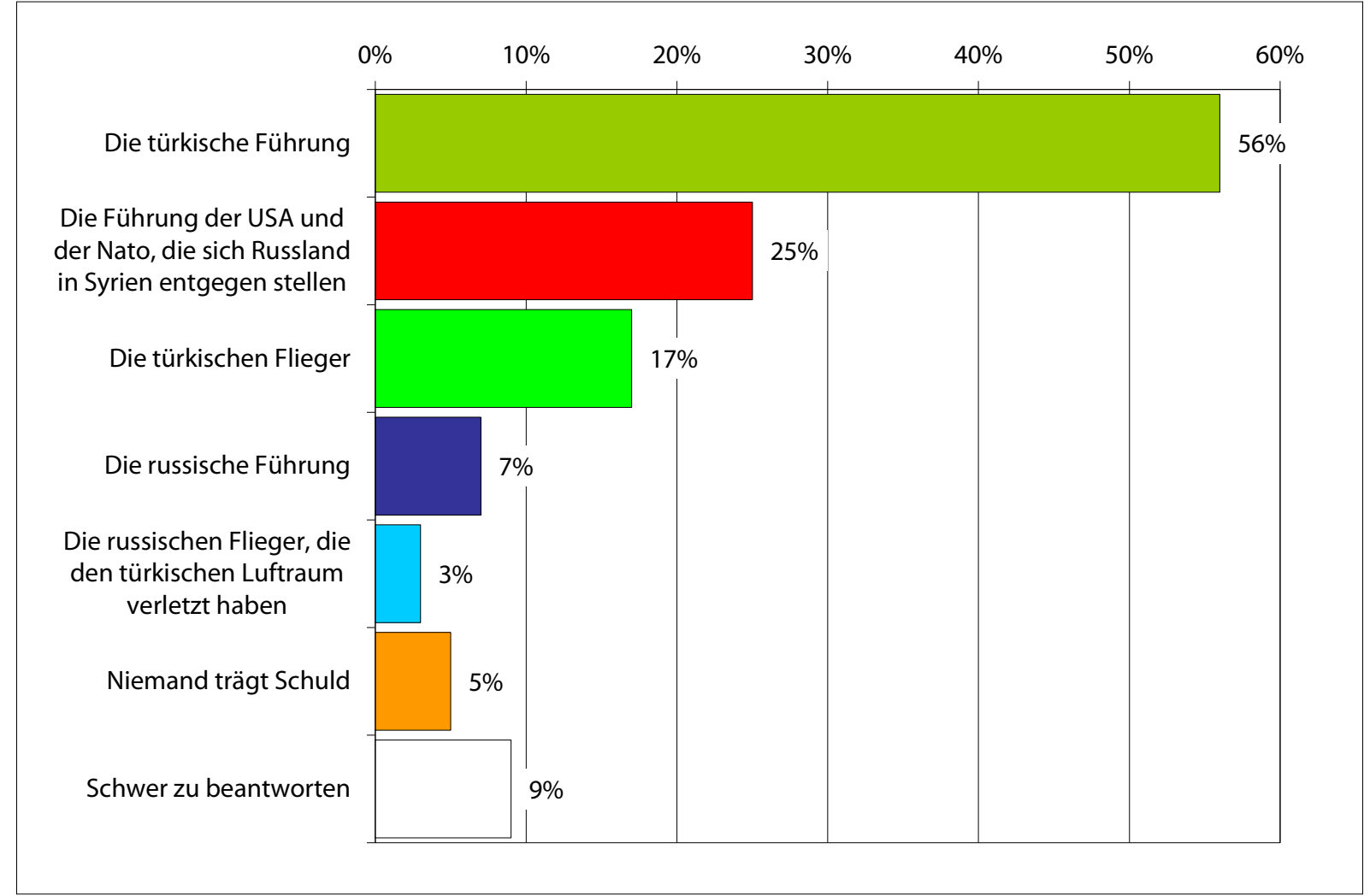

Quelle: Umfragen des Lewada-Zentrums vom 18.-21. Dezember 2015, <http://www.levada.ru/2015/12/25/rossiyane-ob-atake-tu retskih-vs/>, 25. Dezember 2015

AUS RUSSISCHEN BLOGS

\section{Zum Zerfall der »Demokratischen Koalition»}

Sergey Medvedev, Berlin / Moskau

"Enttäuschung«, "Ärger« und »Erleichterung « - mit diesen Begriffen reagiert das russische Netz auf den Zerfall der sogenannten »Demokratischen Koalition«, die als gemeinsame Front im September 2016 bei den Dumawahlen antreten wollte. Das bröckelnde Bündnis aus fünf meist nicht registrierten Oppositionsparteien mit "Parnas" an der Spitze ist am 27. April endgültig auseinandergebrochen, nachdem Alexej Nawalnyj, der Vorsitzende der »Fortschrittspartei«, und Wladimir Milow, der Vorsitzende der »Demokratischen Wahl«, ihren Austritt aus der Koalition erklärten. Der Grund ist der Streit zwischen den Koalitionsmitgliedern und dem »Parnas«-Vorsitzenden Michail Kasjanow über die Bedingungen für die Vorwahlen (»prajmeris«) und den ersten Platz auf der Kandidatenliste sowie über die mangelnde Finanzierung des Vorwahlverfahrens.

Die »Demokratische Koalition« war im Frühjahr 2015 auf der Basis von »Parnas« gegründet worden, um bei den Regionalwahlen im September 2015 und den Dumawahlen 2016 gemeinsam antreten zu können. Da die Partei des ehemaligen Ministerpräsidenten Michail Kasjanow die einzige Partei in der Koalition ist, die zu den Wahlen zugelassen ist, mussten die anderen Parteien - die »Fortschrittspartei« von Alexej Nawalnyj, die »Partei 
des 5. Dezember«, die »Libertäre Partei« und die Partei »Demokratische Wahl« - auf die Vereinbarungen mit der Parteispitze von »Parnas« eingehen. Im Dezember war beschlossen worden, die Plätze auf der Kandidatenliste der Koalition entsprechend der Ergebnisse eines offenen Vorwahlverfahrens zu besetzen. Die einzige Vorbedingung war: Angesichts der besonderen Rolle von »Parnas« in der Koalition wegen ihrer Zulassung zur Wahl sollte Kasjanow bei den Vorwahlen nicht antreten müssen und automatisch den ersten Platz in der Liste bekommen. Diese Entscheidung war unter den Koalitionsmitgliedern und ihren Anhängern von Anfang an stark umstritten.

Am 1. April 2016 zeigte der Staatssender NTW eine Reportage über die Liebesaffäre von Michail Kasjanow und Natalja Pelewina, Parteivorstandsmitglied von »Parnas«. Auf dem Video, das heimlich aufgenommen wurde, ist zu hören, dass sich Kasjanow und Pelewina über die Ansprüche von Alexej Nawalnyj und Ilja Jaschin auf eine Führungsposition bei »Parnas« empören. Obwohl alle Verbündeten aus der Koalition den Film des »PropagandaSenders« kritisierten, wurde er zum Anlass, die Regelung zum ersten Platz auf der Kandidatenliste für den ehemaligen Ministerpräsidenten wieder in Frage zu stellen. Neben der Liebesaffäre wird Kasjanow eine schlechte Organisation des Vorwahlverfahrens und eine unzureichende Finanzierung des Wahlkampfes vorgeworfen.

Laut Umfragen kann »Parnas« nur mit rund einem Prozent der Wählerstimmen rechnen und würde somit an der Fünf-Prozent-Hürde scheitern. Der Zerfall der »Demokratischen Koalition« könnte dazu führen, dass von ihren potenziellen Wählern ein Teil zur Partei "Jabloko« wechselt und viele die Septemberwahl boykottieren. Eine neue demokratische Koalition mit Jabloko ist allerdings auch nicht ausgeschlossen. Medienberichten zufolge führen junge Politiker aus der "Fortschrittspartei«, der die Registrierung durch das Justizministerium aus formellen Gründen mehrmals verweigert wurde, gerade Verhandlungen mit Jabloko über ein gemeinsames Antreten bei den Dumawahlen.

Wie und warum das Bündnis aus fünf Oppositionsparteien zerbrochen ist, schildern Stellungsnahmen einiger ehemaliger Koalitionsmitglieder. Zum erneuten Streit im demokratischen Lager haben sich unter anderem zu Wort gemeldet: der stellvertretende Vorsitzende von Parnas, Ilja Jaschin, der Vorsitzende von Parnas, Michail Kasjanow, der Vorsitzende der »Fortschrittspartei«, Alexej Nawalnyj, der Publizist Anton Krasowskij, der Journalist Kirill Rogow, der Spitzenkandidat von Parnas in Nowosibirsk, Jegor Sawin, das Vorstandsmitglied der Fortschrittspartei Leonid Wolkow und das Mitglied der Partei „Bürgerplattform» Walerij Fedotow.

Jaschin: Ich will nicht die ganze Wahlkampagne über die idiotische Frage beantworten müssen, wer mit wem geschlafen hat

"[...] Ich sage es gleich: Das Privatleben von Kasjanow geht mich nichts an und ich will über niemandes »moralisches Erscheinungsbild« sprechen. Vielmehr habe ich Kasjanow meine menschliche Solidarität ausgesprochen. Ich bin selbst einmal mit versteckter Kamera in einer ähnlichen Situation gefilmt worden. Das Regime setzt solche kriminellen Methoden nicht zum ersten Mal ein.

Gleichzeitig weiß ich sehr genau, dass unsere einzige Chance, die Wahl zu gewinnen, darin besteht, eine Agenda zu formulieren, die für unsere Wähler attraktiv ist. Wir können die Wahl gewinnen, wenn wir die Probleme der Korruption aufgreifen, Putins Diebe ans Tageslicht zerren und die Verhaftung Kadyrows versuchen zu erreichen. Aber unter diesen gegebenen Bedingungen werden wir den ganzen Wahlkampf über idiotische Fragen darüber beantworten müssen, wer mit wem geschlafen hat und wer im Bett über wen geschimpft hat. Somit sind Kasjanows Möglichkeiten als Spitzenkandidat blockiert. [...]«

Ilja Jaschin am 12. April auf Facebook; <https://www.facebook.com/yashin.ilya/posts/1016629981723980>.

\section{Kasjanow: Der Austritt ist ein Fehler}

"Der Druck auf uns war zu erwarten. Wir müssen aber unsere Bewegung fortsetzen. Wenn wir Angst haben weiterzugehen, wird das Regime den Druck noch weiter erhöhen. Ich halte aber die Entscheidung [den Austritt aus der Koalition; "Doschd«] für einen Fehler [...] Wir haben eine grundsätzliche Position. [...] Wir bewegen uns in strenger Übereinstimmung mit dem vereinbarten Verfahren und den Regeln vom Ende des letzten Jahres."

Michail Kasjanow am 27. April im Interview für den TV-Sender "Doschd«; <https://tvrain.ru/news/kasjanov-408304/>.

\section{Nawalnyj: Zu Wahlen, Wählerinteressen und der Demokratischen Koalition}

"[...] Schon Anfang März wurde allen vollkommen klar, dass die Koalition einen großen politischen Fehler begangen hat. Für unsere Anhänger und Wähler sind Vorwahlen, bei denen die Frage über den ersten Platz nicht entschieden wird, einfach nicht interessant. Da kann man nicht drüber hinwegtäuschen; es ist klar, dass der Spitzenplatz das wichtigste in der Wahlkampagne ist; folglich sind wir auf ein völliges Desinteresse an den Vorwahlen der Koalition 
gestoßen. Die Wähler haben sich nicht angemeldet (bisher gibt es wohl keine achttausend davon) und die Debatten werden von niemandem geschaut - die letzten wurden gerade mal von 25 Menschen online angeschaut.

Es gab auch ein technisches Problem: Parnas hat die Aufgabe mit der Website übernommen, auf der man sich für die Vorwahlen anmelden kann, hat es aber überhaupt nicht hingekriegt. Das ist aber eine zweitrangige Frage. Die Menschen würden einen Weg finden, sich anzumelden, wenn sie es wollten.

Fehler können jedem passieren; wichtig ist, sie einzugestehen und zu korrigieren. Man muss sich an den Tisch setzen und besprechen, wie man eine Lösung für die Probleme finden kann, und nicht so tun, als ob nichts passiert wäre.

Ich habe gesehen, dass viele schreiben: „Vereinbarungen, man darf Vereinbarungen nicht brechen«; auch Michail Michailowitsch Kasjanow wiederholt dies ohne Ende.

Ich sage Euch aber Folgendes: Treten wir bei den Wahlen an, um bestimmte Vereinbarungen zu befolgen oder um zu gewinnen? Was ist für uns wichtig: Michail Michailowitsch Kasjanow einen Gefallen zu tun oder die demokratisch orientierten Wähler zu konsolidieren? Politik ist ein lebendiger Prozess. Und das Wahlrennen ist deshalb ein »Rennen«, weil sich alles schnell ändert.

Ungefähr zur selben Zeit, als wir die Vereinbarung über den Spitzenplatz für Kasjanow ohne Vorwahlen trafen, gab es bei den Republikanern in den USA viele Absprachen darüber, dass Jeb Bush ihr Spitzenkandidat wird. Einige Monate sind vergangen und wo ist dieser Jeb Bush jetzt? Er mag ein guter Mensch sein und alle wollen auch ihm etwas Gutes tun; aber bei Wahlen geht es darum, was für die Wähler von Vorteil ist und nicht darum, wie man dem Parteiführer ein Tänzchen tanzt.

Bei der Bildung der Demokratischen Koalition hat es zwei wichtige und felsenfeste Vereinbarungen gegeben: Die Kandidatenlisten werden auf der Grundlage von Parnas gemacht und nur über Vorwahlen gebildet.

Von diesen Vereinbarungen darf man nicht abgehen. Über alles andere kann und soll die Koalition je nach politischer Lage entscheiden.

Genau diese Vereinbarungen haben wir verletzt, indem wir M.M. Kasjanow Sonderrechte eingeräumt haben. Wofür uns die Wähler dann auch ignoriert haben.

Ich habe von Michail Michailowitsch eine sehr gute Meinung. Aufgrund seiner Fachkompetenz wäre er ein ausgezeichneter Abgeordneter und Fraktionsvorsitzender; wir sollen aber im Interesse unserer Anhänger agieren, die »mit den Beinen« abgestimmt haben.

Als Folge haben vier von fünf Mitgliedern der Koalition erklärt, dass wir zu der ursprünglichen Idee zurückkehren sollten: Vorwahlen für die ganze Liste ohne Sonderrechte und Privilegien.

Parnas hat die Entscheidung blockiert [...]. In dieser Situation machen wir - die Fortschrittspartei - das, was wir machen müssen. Wir halten weiterhin Parnas für unseren politischen Verbündeten, erklären aber unseren Rückzug aus der Kandidatenliste von Parnas. [...]"

Alexej Nawalnyj am 28. April aufseinem Blog »navalny.com«; <https://navalny.com/p/4850/>.

\section{Krasowskij: Alles, was Sie über Prinzipien, Konsequenz und Vereinbarungen wissen müssen}

"Wir haben einen Spitzenkandidaten, aber nicht, weil er Parteiführer ist und nicht, weil er zu irgendeiner Nomenklatura gehört, sondern weil Kasjanow der beste Premierminister, der beste Finanzminister war und sich mit den feinen Verknüpfungen und Fallstricken der Bürokraten sehr gut auskennt.« - so Nawalnyj am 11. 12. 2015.

"Wenn der Spitzenkandidat nicht sicher ist, dass er bei den Vorwahlen den ersten Platz erreicht, ist er kein besonders guter Spitzenkandidat.« Nawalnyj am 27.04.2016.

Das ist alles, was Sie über die Prinzipien, Konsequenz und Vereinbarungen wissen müssen«. Anton Krasowskij 27. April 2016 auf Facebook; <https://www.facebook.com/krasovkin/posts/10154280723990809>.

\section{Rogow: Erleichterung}

"Das Gefühl, das sich bei der Nachricht vom Zerfall der demokratischen Koalition einstellte, war ein Gefühl der Erleichterung. Sie erschien mir von Anfang als künstliches Konstrukt, das kein gutes Ende nehmen konnte. Mir hat nicht gefallen, dass Wladimir Ryschkow von dem Erscheinungsbild von "Parnas« abgestoßen wurde. Mit hat absolut nicht gefallen, dass die Konfiguration der Koalition sich so ergab, wie sie dann gekommen ist, wie es mir offensichtlich schien, als Ergebnis des Mordes an Boris Nemzow.

Schließlich stimme ich der Einschätzung von Leonid Wolkow vollkommen zu, dass das Problem von Michail Kasjanow nicht sein idiotischer Spitzenname [gemeint ist "Mischa - 2 prozenta (dt.: "2-Prozent-Micha«), der auf das angeblich korrupte Handeln Kasjanows als Ministerpräsident 2000-2004 anspielt; Anm. d. Red.] oder ähnliches ist, 
sondern vielmehr darin besteht, dass er sich nicht wie ein Oppositionspolitiker, sondern wie ein ehemaliger Premierminister verhält, was unter den aktuellen Umständen den Eindruck eines Blenders schafft.

Die Tatsache ist nämlich die, dass jene, die den Kern der oppositionellen Koalition ausmachen könnten, entweder wegen fabrizierter [Straf]Verfolgung nicht zur Wahl zugelassen sind oder ermordet wurden. Das ist das wahre Bild vor den Wahlen.

In vielen Büchern für Politikwissenschaft ist zu lesen, dass für die Opposition die Strategie teilzunehmen besser ist, als die Strategie nicht teilzunehmen. Es geht aber darüber hinaus darum, dass die Opposition in der Wirklichkeit jedes Mal dieses Dilemma auflösen und jene Art der Teilnahme suchen muss, auf die das Regime nicht vorbereitet ist. Kirill Rogow am 28. April auf Facebook; <https://www.facebook.com/kirill.rogov.39/posts/1232448793439405>.

\section{Sawin: Michail Kasjanow ist nur der Anlass}

"Ich glaube, die Demarche von Nawalnyj und Milow resultiert aus der Angst vor der Verantwortung für das Ergebnis. Bei den Vorwahlen ist etwas schief gelaufen: Die anfängliche Teilnehmerzahl [bei den Vorwahlen] unterscheidet sich sehr von der Realität. Um Vorwürfen auszuweichen, wurde beschlossen, sich zurückzuziehen. Und der Anlass ist MMK [Michail Kasjanow]. Und es ist nur ein Anlass. Man muss sich ja nur mal die Frage stellen: Hätte es die Demarche auch bei 100.000 Teilnehmern an den Vorwahlen gegeben?»

Jegor Sawin am 28. April auf Facebook; <https://www.facebook.com/egor.savin.71/posts/10201823650708593>.

\section{Wolkow: Es geht nicht um Kasjanow selbst, sondern um seinen Ruf}

"[...] Ich habe viele Anhänger getroffen, habe viele Kommentare gelesen. Die Freiwilligen schreiben: »Ich kann mir nicht vorstellen, wie ich auf der Straße für Kasjanow werbe«. Jene Freiwilligen, mit denen wir durch Feuer und Wasser gegangen sind, Moskau und Kostroma durchgemacht haben. Die Anhänger schreiben: »Ich kann mir nicht vorstellen, für Kasjanows Wahlkampf Geld zu spenden«. Ich habe die Frage auf mich übertragen und musste feststellen: Ich könnte es auch nicht. Ich habe mir die Soziologie, die Umfragen angesehen, habe sie gedreht und gewendet: Drei Viertel der Anhänger unserer Werte sind nicht bereit, eine Partei mit Kasjanow an der Spitze zu wählen. In vielerlei Hinsicht geht es nicht um Kasjanow, sondern um sein Image: Dieses Image, das durch die Putin-Medien künstlich geschaffen wurde, ist um Längen schlechter als Michail Michailowitsch. Aber es existiert und wird ein realer Faktor des politischen Kampfes bleiben.

Und das Wichtigste: Ich bin von dieser Idee nicht begeistert und glaube nicht, dass eine solche Kandidatenliste die Chance hat, mehr als 1,5-2\% zu erreichen. Deswegen kann ich Euch nicht aufrichtig dazu aufrufen, Geld für den Wahlkampf zu geben, eure ganze Zeit dafür aufzubringen, Flugblätter zu drucken und zu verteilen, im Callcenter zu sitzen... Es wäre schlichtweg nicht die Wahrheit und würde nicht funktionieren. Nur auf der Liste zu stehen (selbst auf einem der oberen Plätze), das ist es nicht, das wäre nicht interessant.

Ja, sehr schade. Ja, ich hätte gerne an diesen Wahlen aufs Aktivste teilgenommen; ich bin überzeugt, dass ich ein sehr guter Abgeordneter der Staatsduma sein könnte. Nun, daraus wird wohl nichts: Ok, dann werde ich mich mit dem beschäftigen, was ich besser kann, und werde zu einem sehr guten Verteidiger des Internets.

Und überhaupt: seid nicht allzu traurig; in Russland wird das Regime nicht durch Wahlergebnisse abgelöst." Leonid Wolkow am 28. April auf Facebook; <https://www.facebook.com/leonid.m.volkov/posts/1083258721696760>.

\section{Fedotow: Nawalnyj muss Kompromissbereitschaft lernen}

"Bevor er Nummer eins sein will, sollte Nawalnyj erst einmal lernen, Nummer zwei zu sein. Und auch Nummer zehn. Für einen Menschen, der gegen Autoritarismus und andere Formen des Egozentrismus kämpft, wäre dies eine nützliche Fertigkeit. Einen Pornofilm von »NTW« zu benutzen, um eine Attacke gegen Gleichgesinnte zu starten, deren Qualitäten man erst gestern noch so hochgehalten hat, ist dreckig. Als NTW über den »Kirower Forst« berichtete [in einem umstrittenen Strafverfahren war Alexej Nawalnyj 2013 wegen Unterschlagung angeklagt und zu fünf Jahren Haft auf Bewährung verurteilt worden; d. Red.], sind wir zur Unterstützung des Angeklagten auf die Straße gegangen und haben keine Intrigen gegen ihn gesponnen, die eine Schwächung seiner Wählbarkeit zum Ziel haben.

Ich bin zutiefst enttäuscht. Von nun an sind Chodorkowskij, Parnas und Jabloko meine politische Orientierung. Die Reihenfolge entspricht dem Kriterium Kompromissbereitschaft und dem der zumindest nach außen gezeigten Bereitschaft, Ansprüche herunterzuschrauben. Ich träume davon, irgendwann eine wahre Geschlossenheit der russischen demokratischen Opposition zu sehen, und kein lästiges Hickhack.» Walerij Fedotow am 28. April auf Facebook; <https://www.facebook.com/fedotov.valeriy/posts/865479613574191>. 


\title{
Konzilianz und Härte - vom Verlust der Putinschen Stabilität
}

\author{
Jens Siegert, Moskau
}

$\mathrm{M}$ itte April stellte sich Wladimir Putin wie jedes Jahr im Fernsehen fast vier Stunden lang wohl ausgewählten Fragen des Volkes. Putin wirkte in der "Direkter Draht" genannten Sendung viel verbindlicher als im Jahr zuvor. Er bemühte sich auf die Nöte der Fragenden einzugehen und wusste auf (fast) jede Frage eine (meist beruhigende) Antwort. Das war ein deutlicher Unterschied zum »Direkten Draht « von vor einem Jahr. Seinerzeit hatte sich der russische Präsident weit ungeduldiger gezeigt und war konkreten Antworten zu innenpolitischen Fragen immer wieder ausgewichen. Zur Außenund Weltpolitik hatte er dagegen ausführlich und meist scharf räsoniert. Trotz (oder vielleicht wegen) eines konzentrierteren Putins wollte aber dieses Jahr echter Drive nicht so recht aufkommen. Vermutlich hängt das auch mit seiner vor dem Hintergrund der turbulenten Vorjahre ein wenig langweilig wirkenden Hauptbotschaft zusammen: Stabilität und Vertrauen in die eigene Kraft. Vor allem an Ersterem zweifeln mittlerweile immer mehr Menschen in Russland.

Dabei sind beide, Stabilität und die Kraft des russischen Staates, nichts Neues für Putin. Im Grunde kann man sie als Motto seiner gesamten Präsidentschaft sehen. 2000, gerade an die Macht gekommen, versprach der junge, gesunde, energische, ja im Zweiten Tschetschenienkrieg gar brutale, neue Präsident fast schon körperlich ja genau das: Den Staat zu stärken und die im historischen Vergleich unsicheren Lebensumstände der 1990 er Jahre zu stabilisieren. Die Sowjetunion mag langweilig gewesen sein und in ihren letzten beiden Jahrzehnten stagniert haben, aber das Leben war äußerst stabil. Besonders daran erinnerten sich die Menschen nach zehn Jahren ständiger Veränderungen und einem beispiellosen Niedergang der Wirtschaft unter Jelzin.

Putin gelang es, in den Augen der allermeisten Menschen in Russland (und auch außerhalb des Landes) schnell und sehr gut, eine Wende herbei zu führen. Seine Umfragewerte schossen schon in den ersten Monaten in bis dahin (bei Jelzin) ungeahnte Höhen. Mit der Wirtschaft ging es rasant aufwärts. Kaum jemand stellte seinerzeit die Frage, ob dieser Erfolg mehr Putins Können oder vor allem dem Glück der schon Ende der 1990er Jahre einsetzenden Ölhausse zu verdanken war. Seinen Staat präsentierte der neue Präsident (vor allem in Tschetschenien) als entschieden und mit neuer Entschlossenheit.

Für seine zweite Amtszeit von 2004 bis 2008 versprach Putin, die Stabilität und die Kraft des Landes auszubauen. Weiter halfen vor allem die sprudelnden Öl-

und Gaseinnahmen, dieses Versprechen zu erfüllen. Aus heutiger Sicht waren das die goldenen Jahre in Putins Präsidentschaft. Die Einkommen der Menschen stiegen und auch international zählte das Wirtschaftswunderland Russland plötzlich zu den Aufsteigern. Folgerichtig stand das Versprechen am Übergang zu Interimspräsident Medwedjew ebenfalls unter dem Motto Stabilität. Medwedjew sollte fortsetzen, was Putin begonnen hatte. Und damit das auch gelingt (und alle daran glauben), blieb Putin, wenn auch als formal Zweiter, als Ministerpräsident, der eigentliche Herrscher im Lande.

Allerdings machte die 2008/2009 einsetzende Wirtschaftskrise einen dicken Strich durch diese Stabilitätsverlängerungsrechnung. Medwedjew versuchte mit einem Modernisierungsdiskurs gegenzusteuern. Damit rief er einerseits zwar beim großstädtischeren, gebildeteren und mobileren Teil der Bevölkerung Hoffnung auf Änderung (in ihren Augen: auf Besserung) hervor. Auf der anderen Seite löste die Aussicht auf Veränderung bei einer Mehrheit aber (wenn auch vorerst leise) Zweifel am Stabilitätsversprechen aus. Aus Medwedews »Modernisierung" wurde nichts. Nach vier Jahren kehrte Putin zurück. Die enttäuschte Hoffnung im liberalen Teil der Bevölkerung schlug in die Winterproteste 2011/2012 gegen Wahlfälschung und Putins Rückkehr um. Der antwortete mit einer präventiven nationalkonservativen Konterrevolution und einer nationalistischen Mobilisierung der Bevölkerung, mit der Krimannexion, dem (militärischen) Angriff auf die Ukraine und der geopolitischen Konfrontation mit dem Westen als deren Grundelemente. Beides trieb seine Umfragewerte in bis dahin ungesehene Höhen, war also, aus Sicht der Herrschaftssicherung, sehr erfolgreich.

Die Mobilisierung von Gesellschaften ist aber immer ein Spiel mit dem Feuer oder, um ein anderes Bild zu nehmen, es ist wie Poker: Man muss, egal wie die Karten sind, den Einsatz immer weiter erhöhen, um im Spiel zu bleiben. Wenn man nicht mehr mitgeht oder nicht mehr mitgehen kann, ist das Spiel schnell vorbei. Es gibt nur die Stabilität des rollenden Rades. Zwar konnte die Mobilisierung den wirtschaftlichen Aufschwung (vorerst) als Stabilitätsanker ersetzen, nachhaltig ist die sie begleitende Erzählung von der Besonderheit, der Größe und der Gefährdung des Landes aber kaum. So steht Putin spätestens seit 2008, seit dem Beginn des Endes der Ölhausse, immer wieder vor der Wahl zwischen zwei Formen von Instabilität, während er gleichzeitig dem Volk Stabilität verspricht und die meisten Menschen in Russland nichts mehr ersehnen als eben diese Stabilität. 
Was diese Wahl heißt, lässt sich an zwei politischen Ereignissen in Russland Mitte April recht gut illustrieren. In ein und derselben Woche folgte einerseits der für eine moderat liberale Politik und wirtschaftliche Reformen stehende ehemalige Finanzminister Alexej Kudrin dem schon längeren Werben Putins und kehrte de facto in den Staatsdienst zurück, indem er Direktor des von der Regierung finanzierten "Instituts für Strategische Studien" wurde. Auf der anderen Seite veröffentlichte der sehr mächtige Leiter des sehr mächtigen Staatlichen Strafermittlungskomitees Alexander Bastrykin einen langen Artikel in der Wochenzeitschrift "KommersantWlast«, der einen detaillierten Maßnahmenkatalog zur endgültigen Umwandlung Russlands in eine Diktatur ohne Wenn und Aber enthielt (ich habe darüber bereits kurz in den vorigen Notizen geschrieben: <http://russ land.boellblog.org/2016/04/19/hybrider-krieg/>).

Das war Mitte April. Auch international gibt es gemischte Signale. Auf der einen Seite legen sich die russische Luftwaffe in Syrien und die russische Diplomatie an allen Fronten ins Zeug, um das Verhältnis zur NATO (und damit zum sanktionierenden Westen) zu verbessern. Sie tun das durchaus mit gewissem Erfolg. Ende April tagte erstmals seit der Krimannexion der NATO-Russlandrat. Zwar blieben die Positionen unvereinbar, aber immerhin saß man wieder ganz offiziell zusammen. Das wurde in Russland, trotz der weiterbestehenden Sanktionen, als ein erster institutioneller Schritt aufeinander zu interpretiert. Gleichzeitig aber gab es eine ganze Serie von aggressiven Vorbeiflügen russischer Kampfflugzeuge an NATO-Schiffen wie der »USS Donald Cook« in der Ostsee oder gefährliche Annäherungen an Kampfflugzeuge von NATO-Ländern. Der Effekt der Annäherung, wenn es ihn denn gegeben hat, verflog im Nu.

Auch hier zeigt sich das Dilemma der internen Mobilisierung. Annäherung ist langweilig und kommt schnell in den Ruch von Anpassung oder gar Schwäche, wenn nicht gleichzeitig die eigene Stärke, die Möglichkeit eines "wir können auch anders" immer wieder deutlich gezeigt wird. Die ihrer selbst unsichere Führung kann Nähe nur bei gleichzeitiger Abgrenzung und demonstrativem Zeigen von (tatsächlicher oder angeblicher) Stärke ertragen. Außenpolitik hat dabei in jüngster Zeit Innenpolitik als Legitimationsmittel der politischen Herrschaft fast völlig ersetzt.

Das erklärt auch, warum sich Putin seit 2014 fast völlig aus der Innenpolitik zurückgezogen hatte. Er reagierte (siehe "Direkter Draht" vom Vorjahr) gereizt, wenn er sich mit ihr befassen musste. Dieses Feld überließ er der Regierung unter Ministerpräsident Dmitrij Medwedjew und kümmerte sich lieber um die große Weltpolitik. In den Augen eines großen Teils der Bevöl- kerung durchaus mit Erfolg. Zur Wirtschaftsentwicklung fand Putin in dieser Zeit kaum mehr als beruhigende Worte. Ja, das Land sei in der Krise, erklärte er mehrfach, aber das Tal durchschritten und baldige Erholung in Sicht. Doch die Erholung lässt auf sich warten. Wahrscheinlich gibt Putin auch deshalb nun wieder, wie beim »Direkten Draht«, verstärkt den Kümmerer.

Doch es ist ein Kümmern, das (zumindest bisher) kaum mehr als Schmerzlinderung und Tröstung bereithält. Alle Versuche, sich mit anderen großen Ölförderländern auf eine Deckelung der Ölproduktion zu verständigen, sind (bisher) gescheitert. Wegen der Aufhebung der Sanktionen gegen den Iran und erheblichen Interessensunterscheiden mit Saudi Arabien (nicht nur in Syrien) sieht es auch nicht so aus, als könne sich das in nächster Zeit ändern. Erneut in erheblichem Maße steigende Einnahmen aus dem Öl- und Gasexport (und das wäre nötig) sind also nicht zu erwarten. Strukturelle Reformen der russischen Wirtschaft bleiben Stückwerk, vor allem, weil sie, hier herrscht große Einigkeit unter Experten, ohne politische Reformen kaum ausreichend vorankommen werden.

Mitte April veröffentlichte die Staatliche Moskauer Hochschule für Wirtschaft (HSE) die Ergebnisse einer Umfrage unter führenden Wirtschaftsexperten. 90 Prozent der Befragten hielten eine Fortsetzung der bisherigen Wirtschaftspolitik für aussichtslos; mehr als die Hälfte sagte für diesen Fall einen weiteren Abschwung voraus; ein weiteres Drittel eine Stagnation. 70 Prozent nannten beschleunigte Reformen als den besten Weg aus dieser Situation, während aber gleichzeitig kaum jemand daran glaubt, dass sie bald kommen werden. (<http://polit.ru/article/2016/04/21/hse/>)

Angesichts dieser Bewertung stellen sich viele Beobachter die Frage, was Alexej Kudrin ausgerechnet jetzt dazu bewogen haben könnte, in Putins Dienst zurück zu kehren. Ende April wurde er zusätzlich zu seinem Institutsdirektorenposten auch zum stellvertretenden Vorsitzenden des präsidialen Wirtschaftsrats ernannt. Dem Rat sitzt Putin selbst vor. Weitere Stellvertreter sind Wirtschaftsminister Ulukajew und Wirtschaftsberater Belousow. Außerdem versammelt der Rat so ziemlich alle, die in Russland wirtschaftspolitischen Rang und Namen haben. Kaum wieder in Amt, klang Kudrin erheblich verbindlicher als zuvor. Es sei alles gar nicht so schlimm und er könne sich Besserung auch ohne Reform vorstellen. Damit steht er aber, siehe die HSEUmfrage oben, ziemlich allein da.

Diese Konfusion zerstört langsam die Illusion von Stabilität auch in der Bevölkerung. Nach einer aktuellen Umfrage des Lewada-Zentrums sehen nur noch 2 Prozent der Befragten die Lage im Land als »stabil» an; 41 Prozent sagen, es gebe »zeitweise Schwierigkeiten«; 14 
Prozent glauben; Russland befinde sich in einer »Phase der Stagnation«; 17 Prozent fürchten eine »kommende Krise», während 16 Prozent ein sich "vertiefendes Chaos" ausmachen. (<http://www.levada.ru/2016/04/20/obyaza nnosti-grazhdan-i-gosudarstva-2/>).

Zwar bleiben Putins Umfragewerte hoch, aber das Vertrauen in ihn ist, auch im Zusammenhang mit der Veröffentlichung der sogenannten PanamaDokumente, wieder auf Vor-Krim-Niveau gesunken. Mehr als die Hälfte der Befragten glauben, dass Putin »seine Macht missbraucht«. Gar knapp 60 Prozent halten ihn für die Korruption in der Staatsführung für verantwortlich. (<http://www.levada.ru/2016/04/27/ rassledovanie-o-panamskih-offshorah/>)

Diese (wenn auch erst beginnende und vielleicht noch aufzuhaltende) Erosion von Putins Popularität führt auch zu Nervosität in der zweiten Kremlreihe. Nicht die liberale Opposition wird dort gefürchtet, sondern möglicherweise weiter steigender Volksunwille. Die im oben bereits erwähnten Zeitschriftenartikel des Chefs des Strafermittlungskomitees Bastrykin geforderten verschärften Kontroll- und Repressionsmaßnahmen des Staates dürften mindestens ebenso, wenn nicht gar in erster Linie in diese Richtung gehen. Auch die Bildung einer Putin direkt unterstellten Nationalgarde aus mehreren Hunderttausend meist wohltrainierter Spezialeinheiten sind vor allem als Präventivmaßnahmen gegen aufkommenden Volksunwillen zu verstehen.

Andererseits lässt sich Bastrykins öffentliche Demarche auch anders interpretieren. Schon Balzac, so die Politologin Jekaterina Schulman, habe geschrieben, "glückliche Frauen« führten kein öffentliches Leben. Ähnliches gelte in Russland für erfolgreiche Leute aus dem Sicherheitsapparat. Bastrykins Aufruf, die Schrauben anzuziehen, könne daher auch als Aufschrei von jemandem interpretiert werden, der Macht und Einfluss zu verlieren drohe.

Wie dem auch sei, scheint es so, als könne (oder wolle) sich Putin erneut nicht zwischen den beiden Polen seiner Präsidentschaft entscheiden. Erneut versucht er Druck auf die (ohnehin uneinige) Opposition mit Großzügigkeiten aus dem (allerdings schwindenden) staatlichen Füllhorn zu verbinden. Die schon gewohnte Begleitmusik bildet heftige (meist antiwestliche) Propaganda. Putin zeigt, wie gewohnt, abwechselnd Konzilianz und Härte. Man kann es aber auch, wie der in Oslo lebende und aus Russland stammende Politologe Pawel Bajew, härter formulieren: »Putin can neither reinvent this corrupt rent-seeking regime as a police state, nor as a developmental semi-democracy." (<http://www.jamestown.org/programs/edm/single/?tx ttnews\%5Btt_news\%5D=45354\&tx_ttnews\%5Bback Pid\%5D=27\&cHash=d11e27aef9bf03c3e9e26aaa101e 75c7\#.VyPQ8UGa8uS>)

Vor allem international wird die Überraschung über seinen nächsten unerwarteten Schritt (inzwischen so etwas wie sein Markenzeichen) oft als (zumindest) taktischer Erfolg wahrgenommen. Tatsächlich aber erfordert jeder aggressive Schritt nach außen mehr Repressionen nach innen und umgekehrt. Während Putins Politik bisher in vielem den geometrischen Linien und Formen eines Kandinsky-Bildes glich, so nähert sie sich zunehmend den verwirrenden Verschlingungen der späten Werke des Amerikaners Jackson Pollack an. Solange es trotzdem wirtschaftlich voran ging, war das kein Problem. Doch jetzt geht es mit dem Land nach unten.

Diesen und andere Texte finden Sie aufJens Siegerts Russlandblog <http://russland.boellblog.org/>.

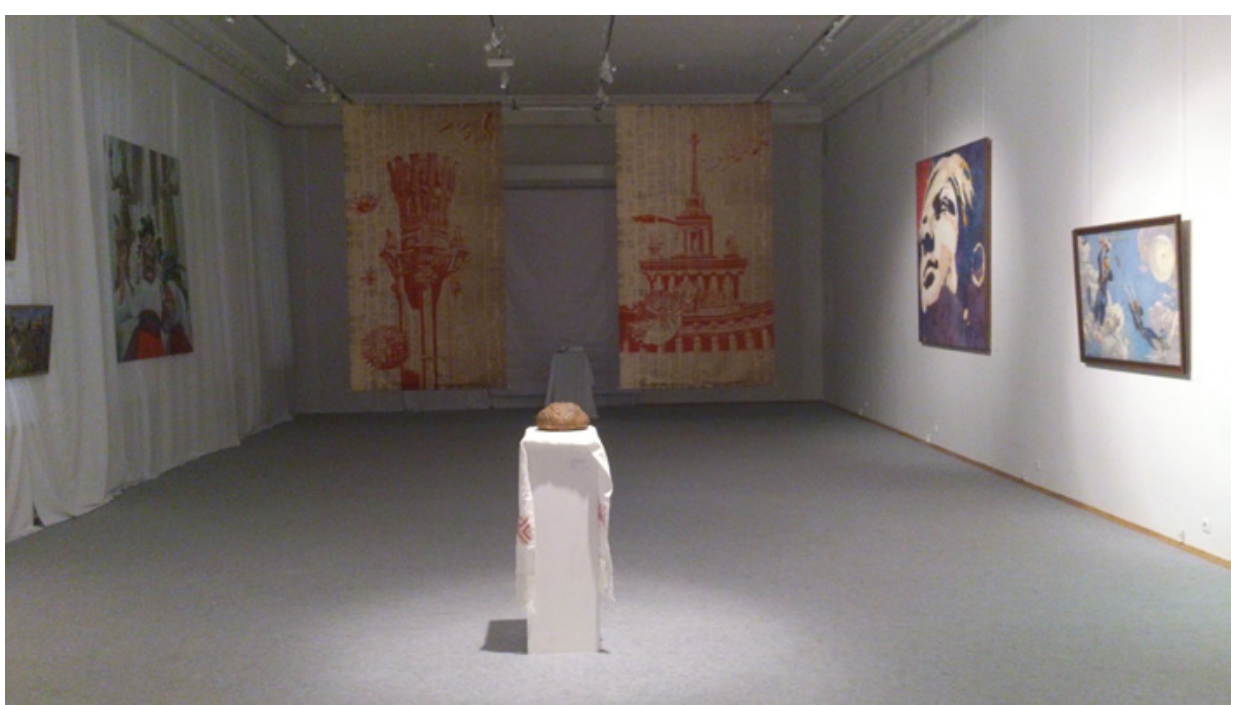

Abbildung 1: Ausstellung im Staatlichen Zentralmuseum für Zeitgeschichte Russlands (Gosudarstwennyj zentralnyj musej sowremennoj istorii Rossii), Moskau, Oktober 2015

Foto: hhs 


\section{Panama Papers}

Grafik 8: Haben Sie von dem »Panama Skandal« gehört und interessiert es Sie?

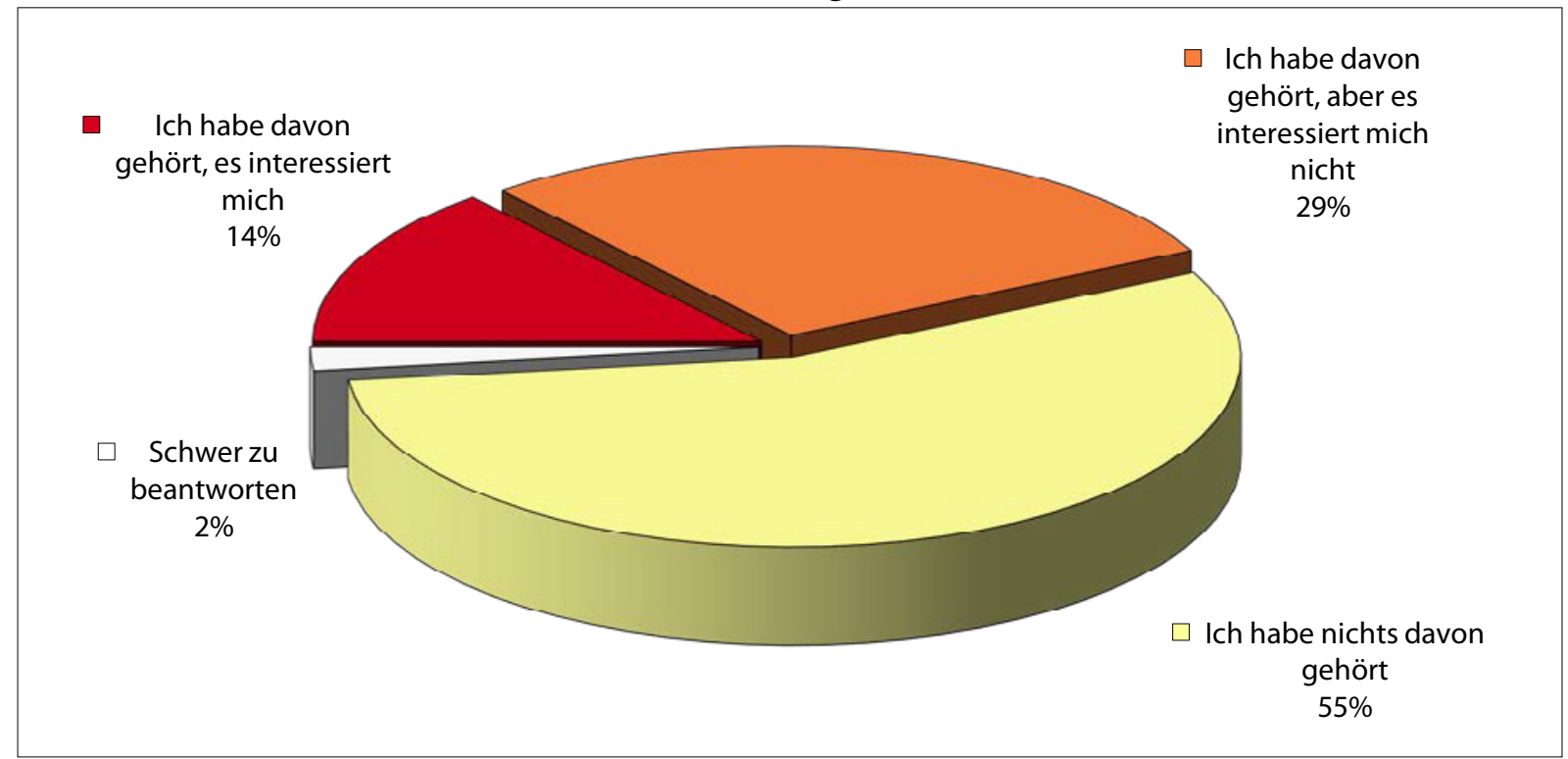

Umfragen des Lewada-Zentrums vom 22.-25. April 2016, <http://www.levada.ru/2016/04/27/rassledovanie-o-panamskih-offsho $\underline{\mathrm{rah} />, 28 .}$. April 2016

Grafik 9: Hat Sie die Information darüber, dass sich unter den russischen Unternehmern mit Offshore-Konten enge Freunde von Putin befinden, überrascht oder nicht überrascht?

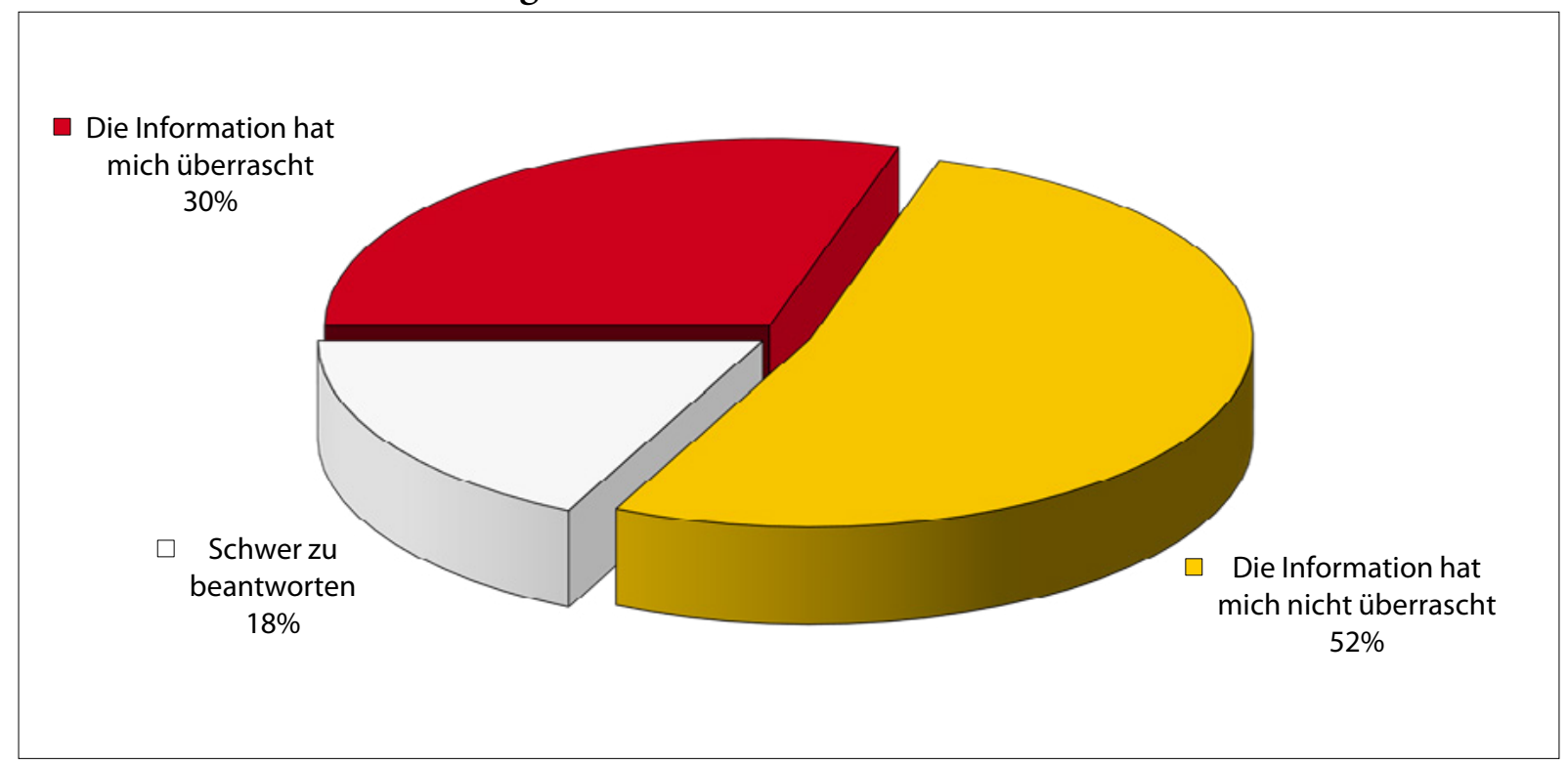

Umfragen des Lewada-Zentrums vom 22.-25. April 2016, <http://www.levada.ru/2016/04/27/rassledovanie-o-panamskih-offsho $\underline{\mathrm{rah} />, 28 .}$. April 2016 
Grafik 10: Wirft die Information einen Schatten auf Präsident Putin oder berührt sie ihn nicht persönlich?

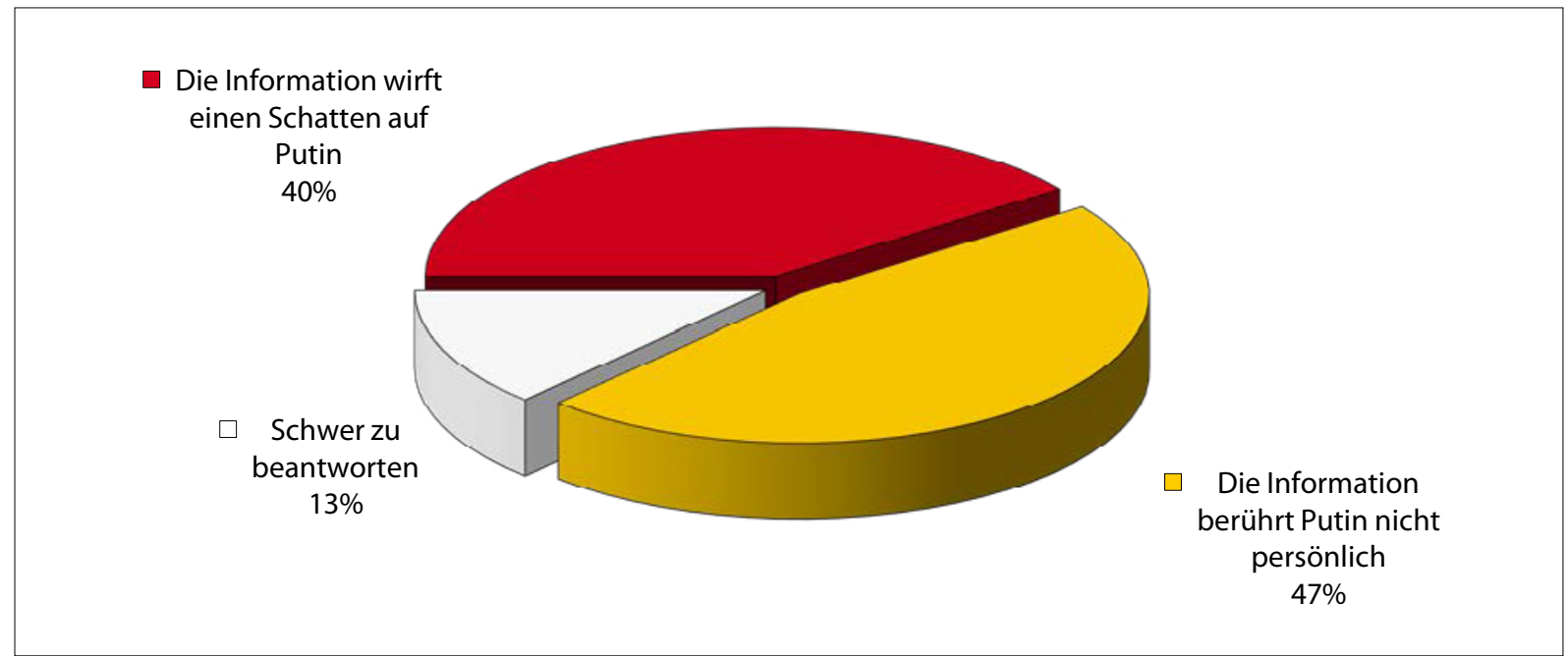

Umfragen des Lewada-Zentrums vom 22.-25. April 2016, <http://www.levada.ru/2016/04/27/rassledovanie-o-panamskih-offsho

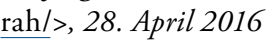

\section{Grafik 11: Mit welchem Ziel wurde das Material in erster Linie publiziert? (eine Antwort)}

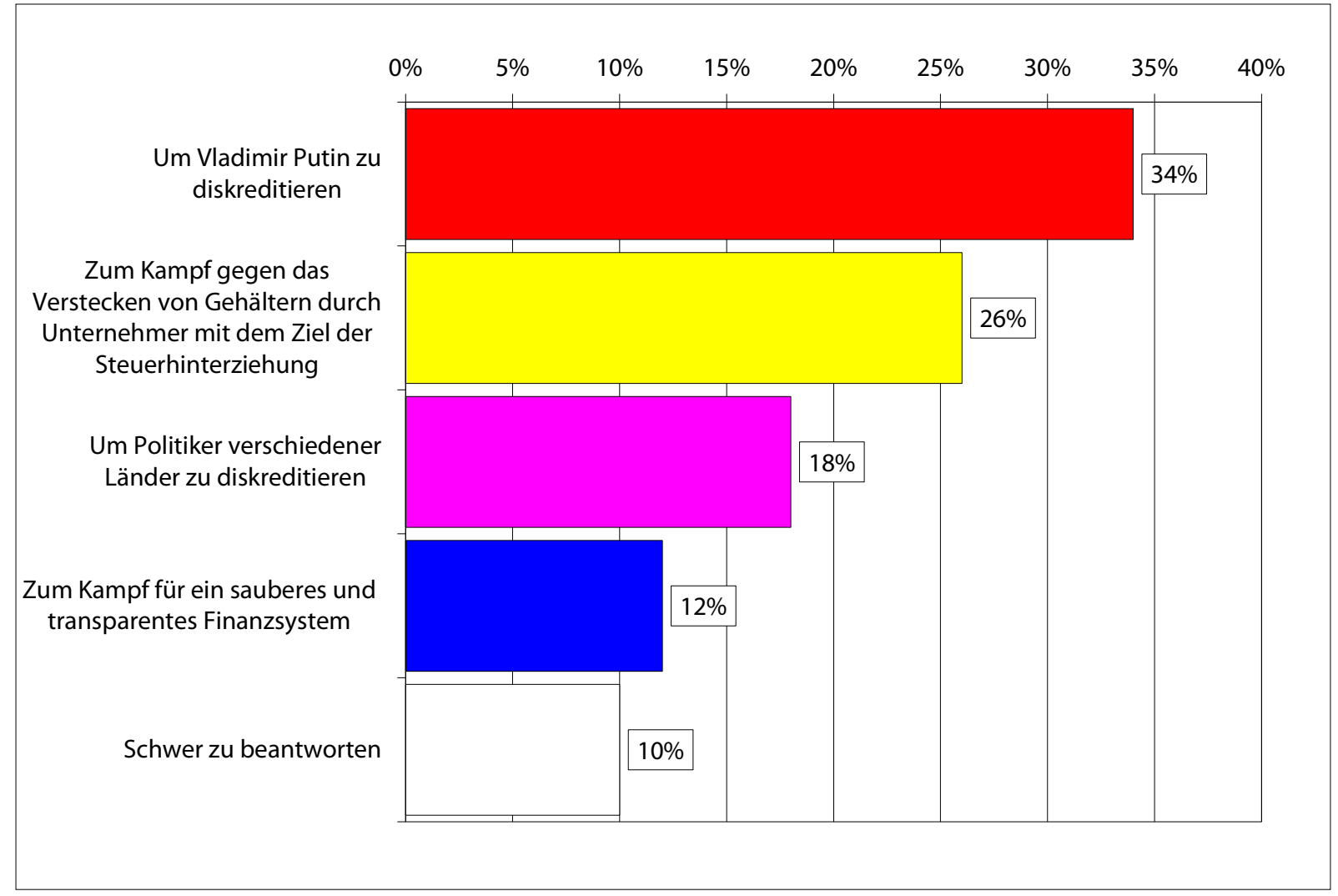

Umfragen des Lewada-Zentrums vom 22.-25. April 2016, <http://www.levada.ru/2016/04/27/rassledovanie-o-panamskih-offsho

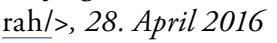




\section{Der »Direkte Draht»}

Grafik 12: Haben Sie das Gespräch des Präsidenten mit den Bürgern Russlands verfolgt oder nicht verfolgt?

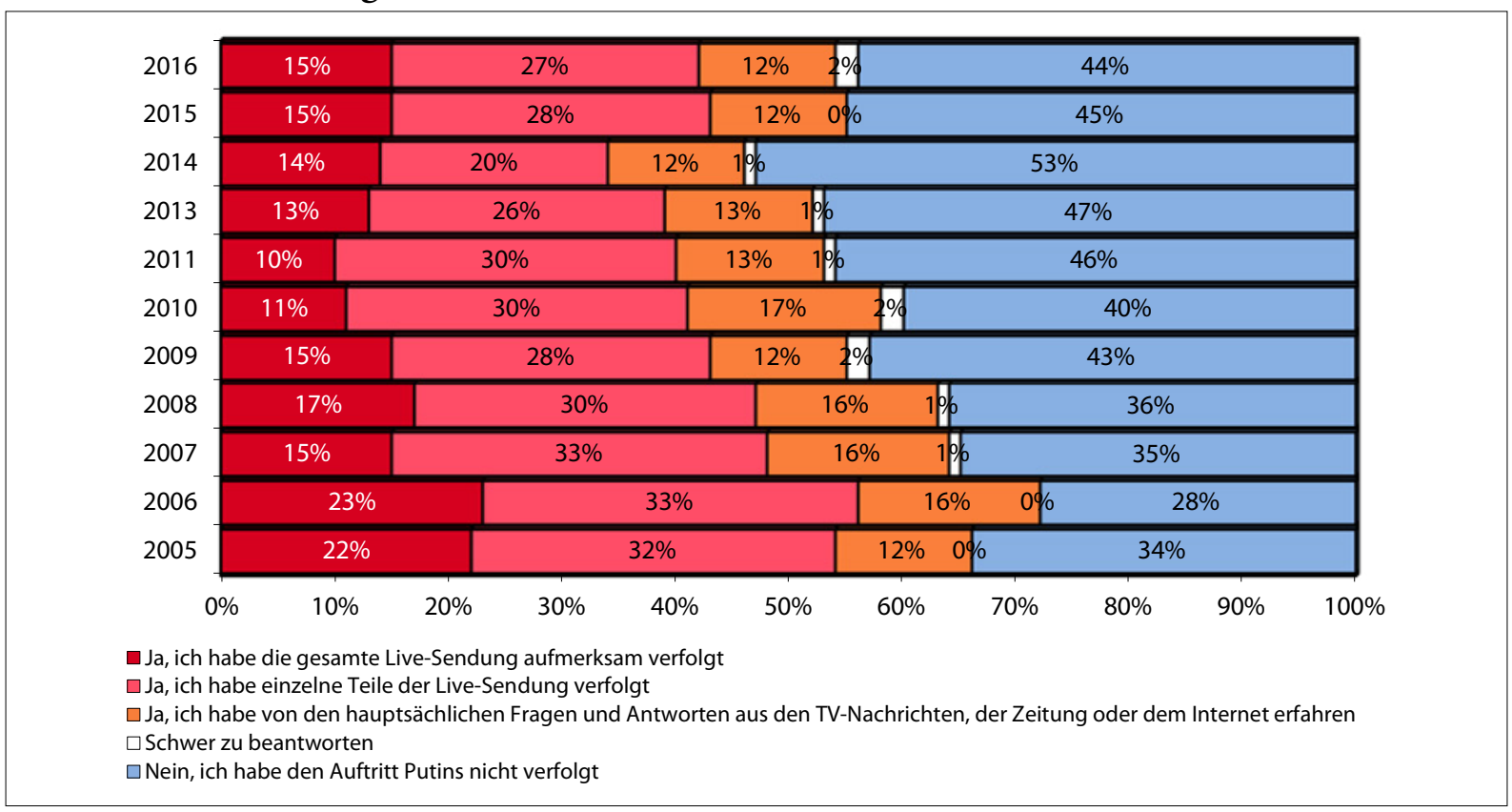

Quelle: Umfragen des WZIOM vom 16.-17. April 2016, <http://wciom.ru/index.php?id=236\&uid=115666>, 22. April 2016

Grafik 13: Welche Antworten auf welche Fragen beim »Direkten Draht« haben Sie am meisten interessiert? (Die zehn meist genannten Antworten 2016; offene Frage, mehrere Antworten möglich)

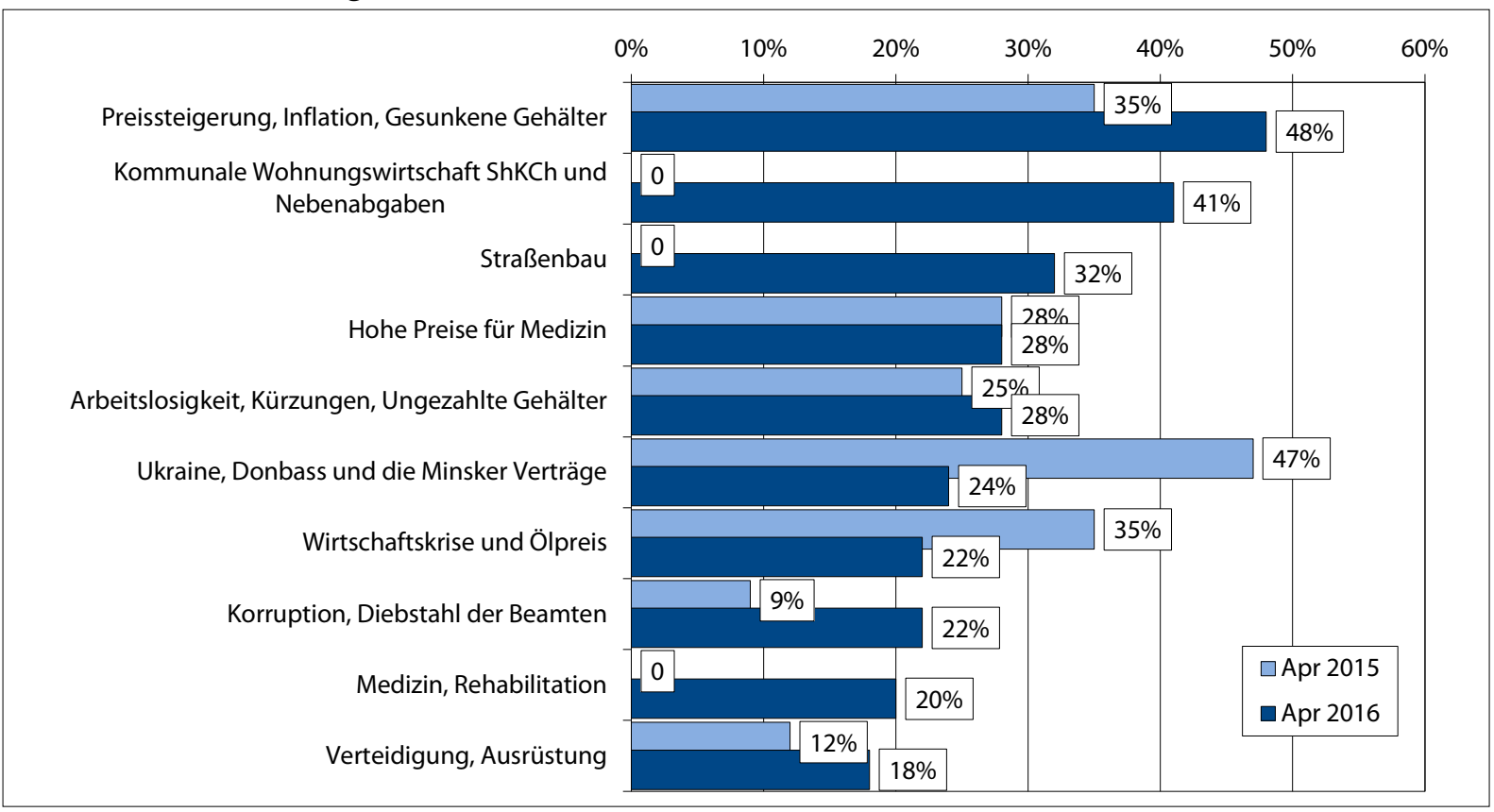

Quelle: Umfragen des Lewada-Zentrums vom 22.-25. April 2016, <http://www.levada.ru/2016/04/28/pryamaya-liniya-vnimaniei-zapomnivshiesya-temy/>, 28. April 2016 


\section{April - 5. Mai 2016}

\begin{tabular}{|c|c|}
\hline 22.4.2016 & $\begin{array}{l}\text { Russland unterzeichnet im UN-Hauptquartier in New York das Pariser Klimaschutz-Abkommen, das im Dezem- } \\
\text { ber } 2015 \text { in der französischen Hauptstadt ausgehandelt worden war. Insgesamt haben Vertreter von mehr als } 170 \\
\text { Ländern das Abkommen unterzeichnet. }\end{array}$ \\
\hline 22.4.2016 & $\begin{array}{l}\text { Die Staatsduma verabschiedet in dritter Lesung ein Gesetz, das es ermöglicht, Abgeordneten wegen } 30 \text { oder mehr } \\
\text { unentschuldigter Fehltage das Mandat zu entziehen. }\end{array}$ \\
\hline 23.4.2016 & $\begin{array}{l}\text { Der Hauptrestaurator des Kulturministeriums Boris Zagarajew muss auf Entscheidung des Gerichts des Mos- } \\
\text { kauer Bezirks Lefortowo für zwei Monate in Haft. Ihm wird vorgeworfen, Finanzmittel des Ministeriums ent- } \\
\text { wendet zu haben. }\end{array}$ \\
\hline 23.4 .2016 & Präsident Wladimir Putin gratuliert den Juden in Russland zum Pessach-Fest. \\
\hline 23.4.2016 & $\begin{array}{l}\text { Ein russischer Tanker gerät auf dem Kaspischen Meer in Brand. Zwei Hubschrauber und mehrere Schiffe sind } \\
\text { bis in den Abend mit den Löscharbeiten beschäftigt. Ein Besatzungsmitglied kommt bei dem Brand ums Leben. }\end{array}$ \\
\hline 25.4 .2016 & $\begin{array}{l}\text { Die USA verlegen zwei Jagdflugzeuge des Typs F-22 Raptor« zur Unterstützung seiner Nato-Bündnispartner in } \\
\text { Osteuropa nach Rumänien. Barack Obama hatte } 2014 \text { im Kontext der Krim-Annexion den östlichen Nato-Part- } \\
\text { nern eine Unterstützung ihrer Verteidigungsfähigkeit zugesagt. }\end{array}$ \\
\hline 26.4.2016 & $\begin{array}{l}\text { Das Oberste Gericht der Krim stuft das Selbstverwaltungsorgan der Krimtataren, den Medschlis, als extremis- } \\
\text { tische Organisation ein und verbietet es. Die Europäische Union kritisiert das Verbot als Angriff auf die Rechte } \\
\text { der Krimtataren. }\end{array}$ \\
\hline 26.4.2016 & $\begin{array}{l}\text { Der Europäische Gerichtshof für Menschenrechte (EGMR) verurteilt Russland dazu, an fünf Personen, die wäh- } \\
\text { rend »individueller Protestwachen« festgenommen und zum Teil verurteilt wurden, Schadensersatz von insgesamt } \\
\text { rund } 35.000 \text { Euro zu zahlen. Der EGMR bewertet das Verhalten der Behörden als Verletzung der Meinungsfreiheit. }\end{array}$ \\
\hline 27.4 .2016 & $\begin{array}{l}\text { Der erste Raketenstart vom neuen russischen Weltraumbahnhof "Wostotschnyj" wird aus technischen Gründen } \\
\text { kurzfristig um einen Tag auf den 28. 4. verschoben. Der Start der Rakete, der für 5.01 Uhr morgens Moskauer } \\
\text { Zeit angesetzt war, wurde 1,5 Minuten vor dem Start automatisch abgebrochen. Präsident Wladimir Putin kri- } \\
\text { tisiert auf einer Sitzung der Staatlichen Kommission für Testflüge von Trägersystemen die mangelnde Kontrolle } \\
\text { und die Schlamperei in technischen Prozessen des Raketenstarts . }\end{array}$ \\
\hline 27.4 .2016 & $\begin{array}{l}\text { Alexej Nawalnyj reicht beim Gericht des Moskauer Sawjolowskij-Bezirks eine Klage gegen die staatliche Medien- } \\
\text { anstalt WGTRK, den stellvertretenden Generaldirektor des Unternehmens Dmitrij Kiseljow und den Journalis- } \\
\text { ten Jewgenij Popow ein. Anlass der Klage ist der Dokumentarfilm "Effekt Braudera» (dt: "Der Browder-Effekt«), } \\
\text { in dem Nawalnyj als bezahlter Agent des britischen Geheimdienstes dargestellt wird. }\end{array}$ \\
\hline 27.4 .2016 & $\begin{array}{l}\text { Der Vorsitzende der »Forschrittspartei«, Alexej Nawalnyj, und der Vorsitzende der Partei »Demokratischen Wahl«, } \\
\text { Wladimir Milow, erklären den Austritt ihrer Parteien aus der »Demokratischen Koalition« mit der Oppositions- } \\
\text { partei »Parnas«. Anlass ist der Streit zwischen den Koalitionsmitgliedern und dem Parnas-Vorsitzenden Michail } \\
\text { Kasjanow über die Bedingungen der Vorwahlen und die Besetzung des Spitzenplatzes der Kandidatenliste. }\end{array}$ \\
\hline 28.4.2016 & $\begin{array}{l}\text { Im zweiten Anlauf gelingt der Start der Trägerrakete „Sojuz-2.1a« vom neuen russischen Weltraumbahnhof »Wos- } \\
\text { totschnyj« im Amur-Gebiet. }\end{array}$ \\
\hline 28.4 .2016 & $\begin{array}{l}\text { Die Preisverleihung des von der Menschenrechtsorganisation "Memorial« veranstalteten Schülerwettbewerbs } \\
\text { "Der Mensch in der Geschichte«, an der auch die Autorin Ljudmila Ulizkaja teilnimmt, wird von der nationa- } \\
\text { listischen Organisation »Nationale Befreiungsbewegung" (NOD) gestört. Rund } 20 \text { Aktivisten der NOD skan- } \\
\text { dieren u. a. "National-Verräter», bewerfen die Teilnehmer mit Eiern und begießen sie mit grüner Flüssigkeit. }\end{array}$ \\
\hline 29.4.2016 & $\begin{array}{l}\text { Vertreter der russischen und der US-amerikanischen militärischen Führung einigen sich auf eine Feuerpause für } \\
\text { einige Landesteile Syriens. In Damaskus soll die Feuerpause } 24 \text { Stunden gelten, in Latakia } 72 \text { Stunden. Für die } \\
\text { syrische Stadt Aleppo wird vorläufig keine Einigung erzielt. }\end{array}$ \\
\hline 29.4.2016 & $\begin{array}{l}\text { Die Nato kündigt an, dass sie eine Verstärkung ihrer militärischen Präsenz in den an Russland angrenzenden Mit- } \\
\text { gliedsstaaten plant. Insgesamt sollen rund } 4.000 \text { Soldaten nach Polen und in die Länder des Baltikums verlegt } \\
\text { werden. Zwei Bataillone sollen durch die USA, und eins jeweils von Deutschland und Großbritannien bereitge- } \\
\text { stellt werden. Die endgültige Entscheidung darüber fällt auf dem Nato-Gipfel in Warschau am 8. und 9. Juli } 2016 .\end{array}$ \\
\hline 30.4 .2016 & $\begin{array}{l}\text { In Moskau gründen Fernfahrer die Gewerkschaft »Vereinigung der Fernfahrer Russlands« (OPR) zum Kampf } \\
\text { gegen das Mautsystem »Platon«. An dem Gründungskongress nahmen rund } 300 \text { LKW-Fahrer aus } 31 \text { Regionen } \\
\text { Russlands teil. }\end{array}$ \\
\hline
\end{tabular}




\begin{tabular}{|c|c|}
\hline 30.4 .2016 & $\begin{array}{l}\text { Präsident Wladimir Putin verleiht vier Männern und einer Frau den Titel »Held der Arbeit der Russischen Föde- } \\
\text { ration«. Ausgezeichnet wurden der Sänger und Duma-Abgeordnete Iosif Kobson, der Leiter des Ölunterneh- } \\
\text { mens »Surgutneftegaz«, Wladimir Bogdanow, der leitende Schäfer der Agargenossenschaft "Zokto-Changil«, } \\
\text { Dalaj Gungajew, der Generaldirektor des Konstrukteursbüros »Nowator«, Pawel Kamnew, und die Lehrerin } \\
\text { Ljudmila Kornilowa. }\end{array}$ \\
\hline 1.5 .2016 & $\begin{array}{l}\text { In Moskau nehmen rund } 100.000 \text { Menschen an dem Demonstrationszug der Gewerkschaften auf dem Roten } \\
\text { Platz teil. Die Gewerkschaften fordern die Erhöhung der Löhne und eine Nichtanhebung des Renteneintrittsalters. }\end{array}$ \\
\hline 1.5.2016 & $\begin{array}{l}\text { Linke und feministische Gruppen demonstrieren in Moskau gegen Homophobie, Sexismus und Faschismus. } \\
\text { Während der Demonstration werden elf Personen festgenommen. }\end{array}$ \\
\hline 1.5 .2016 & $\begin{array}{l}\text { Den »Bikern« der Gruppe "Nachtwölfe« die von Moskau nach Berlin fahren, um dort am 9. Mai den »Tag des } \\
\text { Sieges« zu begehen, wird an der polnischen Grenze die Einreise verweigert. }\end{array}$ \\
\hline 2.5 .2016 & $\begin{array}{l}\text { Mit der Unterzeichnung durch Präsident Wladimir Putin tritt ein Gesetz über die Vergabe von Land an die Bür- } \\
\text { ger im Fernöstlichen Föderalbezirk in Kraft. Gemäß diesem Gesetz können die Bürger einmalig und zunächst } \\
\text { für fünf Jahre einen Hektar Land unentgeltlich erhalten. }\end{array}$ \\
\hline 2.5 .2 & $\begin{array}{l}\text { Mit der Unterzeichnung durch Präsident Wladimir Putin tritt ein Gesetz in Kraft, das den Erhalt einer Aufent- } \\
\text { haltsgenehmigung durch ausländische Flüchtlinge vereinfachen soll. }\end{array}$ \\
\hline 3.5 .2016 & $\begin{array}{l}\text { Außenminister Sergej Lawrow und der UNO-Sonderbeauftragte für Syrien, Staffan de Mistura, führen ange- } \\
\text { sichts der erneut eskalierten Kämpfe in Aleppo Gespräche über eine Waffenruhe in der Stadt. }\end{array}$ \\
\hline 4.5 .2016 & $\begin{array}{l}\text { Verteidigungsminister Sergej Schojgu erklärt, dass Russland an seiner westlichen Landesgrenze drei neue Divi- } \\
\text { sionen zur Abwehr gegen die Nato schaffen will. }\end{array}$ \\
\hline 4.5 .2016 & $\begin{array}{l}\text { Russland und die Ukraine führen anlässlich der kommenden Gedenkwoche übergangsweise ein vereinfachtes } \\
\text { System für den Grenzübertritt von Bewohnern der Grenzregionen ein, damit diese die Gräber ihrer Verwandt- } \\
\text { schaft im jeweils anderen Land besuchen können. }\end{array}$ \\
\hline 5.5 .2016 & Im Gebiet Tjumen werden in mehreren Orten 390 Menschen wegen Hochwasser evakuiert \\
\hline 5.5.2016 & Die russische Zentralbank entzieht den Bankhäusern »Mostransbank» und »El-Bank» die Lizenz. \\
\hline 5.5 .2016 & $\begin{array}{l}\text { Nachdem sich herausgestellt hat, dass der Fehlstart der Trägerrakete »Sojus-2.1.a« am 27.4.2016 auf einen Pro- } \\
\text { duktionsfehler zurückgeht, reicht der für die Entwicklung des Steuerungssystems für Raketentechnik verant- } \\
\text { wortliche Direktor Leonid Schalimow seinen Rücktritt ein. }\end{array}$ \\
\hline
\end{tabular}

Sie können die gesamte Chronik seit 1964 auch auf <http://www.laender-analysen.de/russland/> unter dem Link »Chronik«lesen.

Die Russland-Analysen werden von Mangold Consulting GmbH unterstützt.

\section{MANGOLD}

$\mathrm{C} \circ \mathrm{n} \mathrm{s} u$ । $\mathrm{t}$ i $\mathrm{n} \mathrm{g}$

Herausgeber: Forschungsstelle Osteuropa an der Universität Bremen und Deutsche Gesellschaft für Osteuropakunde

Die Meinungen, die in den Russland-Analysen geäußert werden, geben ausschließlich die Auffassung der Autoren wieder. Abdruck und sonstige publizistische Nutzung sind nach Rücksprache mit der Redaktion gestattet. Redaktion: Hans-Henning Schröder (verantwortlich), Julia Glathe Sprachredaktion: Hartmut Schröder Satz: Matthias Neumann

Russland-Analysen-Layout: Cengiz Kibaroglu, Matthias Neumann und Michael Clemens Alle Ausgaben der Russland-Analysen sind mit Themen- und Autorenindex archiviert unter www.laender-analysen.de Die Russland-Analysen werden im Rahmen eines Lizenzvertrages in das Internetangebot der Bundeszentrale für politische Bildung (www.bpb.de) aufgenommen. Die Russland-Analysen werden im Rahmen der Datenbank World Affairs Online (WAO) ausgewertet und sind im Portal IREON www.ireon-portal.de recherchierbar. ISSN 1613-3390 @ 2016 by Forschungsstelle Osteuropa, Bremen

Forschungsstelle 0steuropa • Publikationsreferat • Klagenfurter Str. 3 • 28359 Bremen • Telefon: + 49 421-218-69600 • Telefax: + 49 421-218-69607 e-mail: publikationsreferat@osteuropa.uni-bremen.de•Internet-Adresse: http://www.laender-analysen.de/russland/ 


\section{Kostenlose E-Mail-Dienste der Forschungsstelle Osteuropa und ihrer Partner auf www.laender-analysen.de}

Die Länder-Analysen bieten regelmäßig im kostenlosen Abonnement kompetente Einschätzungen aktueller politischer, wirtschaftlicher, sozialer und kultureller Entwicklungen in Ostmitteleuropa und der GUS. Sie machen das Wissen, über das die wissenschaftliche Forschung in reichem Maße verfügt, für Politik, Wirtschaft, Medien und die interessierte Öffentlichkeit verfügbar. Autoren sind internationale Fachwissenschaftler und Experten.

Die einzelnen Länder-Analysen werden von der Forschungsstelle Osteuropa an der Universität Bremen und der Deutschen Gesellschaft für Osteuropakunde jeweils mit unterschiedlichen Partnern und Sponsoren herausgegeben. Die Redaktionen der Länder-Analysen bestehen aus Wissenschaftlern mit langjähriger Forschungserfahrung.

Die Länder-Analysen bieten regelmäßig Kurzanalysen zu aktuellen Themen, ergänzt um Grafiken und Tabellen sowie Dokumentationen. Zusätzlich gibt es eine Chronik aktueller Ereignisse. Alle Länder-Analysen sind auch mit Archiv und Indizes online verfügbar unter $<$ www.laender-analysen.de $>$.

\section{Belarus-Analysen}

Erscheinungsweise: zweimonatlich

Abonnement unter: <http://www.laender-analysen.de/belarus/>

\section{Caucasus Analytical Digest}

In englischer Sprache. Erscheinungsweise: monatlich

Abonnement unter: <http://www.css.ethz.ch/en/publications/cad.html>

\section{Polen-Analysen}

Erscheinungsweise: zweimal monatlich

Abonnement unter: <http://www.deutsches-polen-institut.de/newsletter/polen-analysen/>

\section{Russland-Analysen}

Erscheinungsweise: zweiwöchentlich

Abonnement unter: <http://www.laender-analysen.de/russland/>

\section{Russian Analytical Digest}

In englischer Sprache. Erscheinungsweise: zweimal monatlich

Abonnement unter: <http://www.css.ethz.ch/en/publications/rad.html>

\section{Ukraine-Analysen}

Erscheinungsweise: zweimal monatlich

Abonnement unter: <http://www.laender-analysen.de/ukraine/>

\section{Zentralasien-Analysen}

Erscheinungsweise: monatlich

Abonnement unter: <http://www.laender-analysen.de/zentralasien/>

\section{Bibliographische Dienste}

Die Bibliographien informieren über englisch- und deutschsprachige Neuerscheinungen zu Belarus, Russland, Ukraine sowie zu den zentralasiatischen und kaukasischen Staaten. Erfasst werden jeweils die Themenbereiche Politik, Außenpolitik, Wirtschaft und Soziales.

Erscheinungsweise: viermal jährlich

Abonnement unter: <http://www.laender-analysen.de/bibliographies/belarus.php>, <http://www.laender-analysen. de/bibliographies/russia.php>, <http://www.laender-analysen.de/bibliographies/ukraine.php>, <http://www.laenderanalysen.de/bibliographies/caucasus_ca.php> 